\title{
Natural feedstocks for diversity-oriented synthesis: macrolide-like scaffolds from nonactate
}

\author{
Yuliya G. Sumskaya, ${ }^{\text {a }}$ P. Whitney Swain III, ${ }^{\text {b }}$ Stepehen C. Bergmeier, ${ }^{\text {b Mark C. McMills, }}$ \\ Nigel D. Priestley, ${ }^{b}$ and Dennis L. Wright ${ }^{a}$ * \\ ${ }^{a}$ Department of Pharmaceutical Sciences and Department of Chemistry, \\ University of Connecticut, Storrs, CT 06269, USA \\ ${ }^{b}$ Promiliad Biopharma, Alberton, MT 59820, USA \\ E-mail: dennis.wright@uconn.edu
}

Dedicated to Professor William F. Bailey

\begin{abstract}
We have been interested in the application of readily available, fermentation-derived natural products as key building blocks for the preparation of natural product-like libraries rich in structural and stereochemical diversity. In this manuscript we describe the conversion of methyl nonactate, derived from nonactin, to a diversable scaffold characteristic of macrolide natural products. The synthesis features a key ring-closing metathesis reaction to form the macrocycle.
\end{abstract}

Keywords: Natural products, ring-closing metathesis, nonactin, macrolide

\section{Introduction}

The screening of small molecule collections to discover new medicinal chemistry leads is a critical step in the development of many therapeutic agents. ${ }^{1}$ One of the major challenges is developing strategies to deliver new arrays of compounds for screenings that are structurally distinct from those types of compounds commonly found in HTS libraries. It is widely recognized that compounds more reminiscent of natural products are far less prevalent in typical screening libraries. ${ }^{2}$ Whereas natural products are characterized by higher oxygen content, alicyclic systems and abundant stereogenic centers, typical screening compounds are much higher in nitrogen, planar aromatics and extremely limited stereochemical diversity.

We have been interested in developing a strategy for the generation of highly complex compound arrays by integrating the ease and scalability of fermentation with the ability to conduct short, diversification sequences through reliable functional group interconversions. This type of strategy relies on simple fermentation technology to engender a high level of structural and stereochemical complexity in the compound series while routine synthetic transformations introduce key elements of diversity among the library members (Figure 1). 


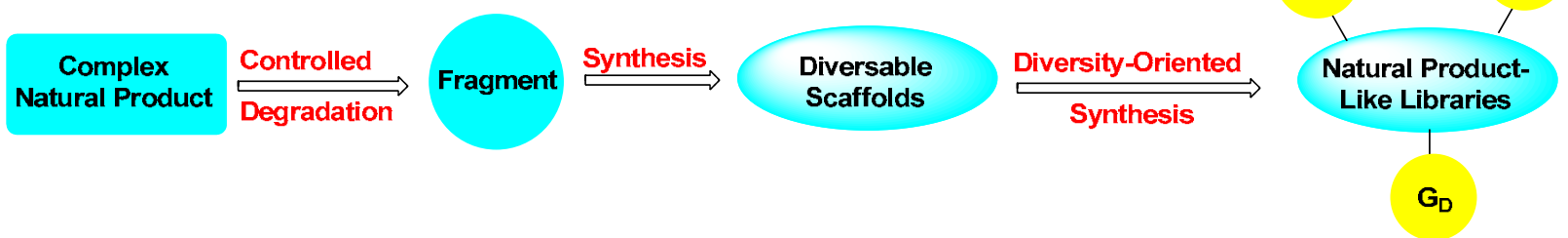

Figure 1. Strategy for the generation of natural product-like libraries $\left(\mathrm{G}_{\mathrm{D}}=\right.$ diverse groups $)$.

A cheap and readily available complex natural product is used as the point of departure for the preparation of key complex fragments that can be parlayed into a small group of diversable scaffolds using classical synthetic methods. A second round of synthesis would then be possible using a diversity-oriented manifold to elaborate the scaffolds into arrays of compounds having more natural product-like features than typical screening libraries.

One natural feedstock that we have found to have great utility in constructing new chemical entities is nonactin, a well known polyketide antibiotic that can be produced in significant quantities from Streptomyces griseus. ${ }^{3}$ Previously, we had developed a method for the degradation of nonactin to give both enantiomers of methyl nonactate $\mathbf{1}$ and $\mathbf{2}$ as well as other isomeric units (Scheme 1). ${ }^{4}$

Nonactin is the parent compound of a group of ionophore antibiotics known as the macrotetrolides. The 32-membered macrocyclic ring of nonactin is composed of two units of $(+)-$ nonactate and two units of (-)-nonactate, alternately arrayed in a macrocycle, so that the molecule has overall S4 symmetry and is achiral. Because nonactin contains an equal number of (+)- and (-)nonactate residues, exhaustive methanolysis of nonactin (Scheme 1) delivers racemic methyl nonactate. ${ }^{5}$ A biotransformation-based kinetic resolution can be initiated by exposure of the racemate to Rhodococcus erythropolis under aerobic conditions to produce methyl (-)-8ketononactate and unreacted methyl $(+)$-nonactate. The ketoester can then be re-reduced by Rhodococcus erythropolis under anaerobic conditions to give methyl (-)-nonactate. Other nonactate scaffolds can be obtained by various stereoselective transformations. It is noteworthy that compounds constructed from these six fragments would yield a library rich in stereochemical and not just structural diversity.

Constructing novel chemotypes from nonactate is a priority as it allows the expansion of these natural product-like libraries into additional areas of unexplored chemical space. This manuscript details our efforts to construct new diversable scaffolds reminiscent of macrolide topology. Macrolide natural products represent a large classification of bioactive secondary metabolites that span a range of activities such as antibiotic, anticancer and immunosupressive properties. Interestingly, it was recently demonstrated that an erythromycin derivative emerges as hit form a library screen for non-peptide LHRH antagonists, thus illustrating that activities distinct from the natural function can emerge with structurally related congeners. ${ }^{6}$ 


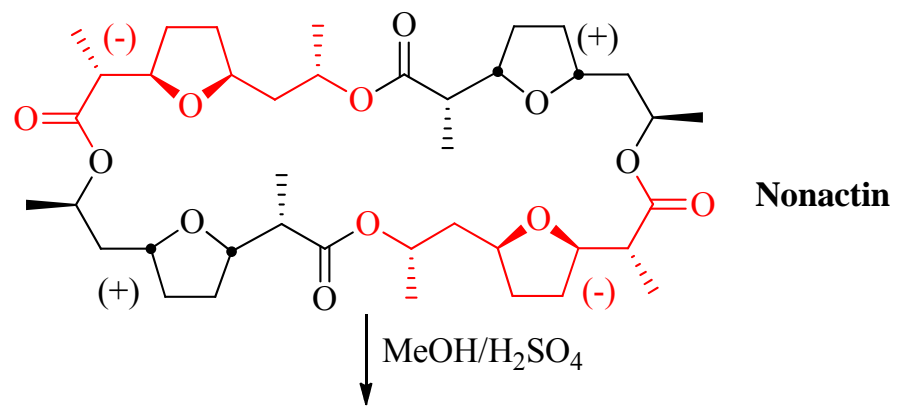<smiles>CC(=O)[C@H](C)[C@H]1CC[C@@H](C[C@@H](C)O)O1</smiles>

(-)-methyl nonactate<smiles>CC(=O)[C@H](C)[C@H]1CC[C@@H](C[C@@H](C)O)O1</smiles>

(+)-methyl nonactate

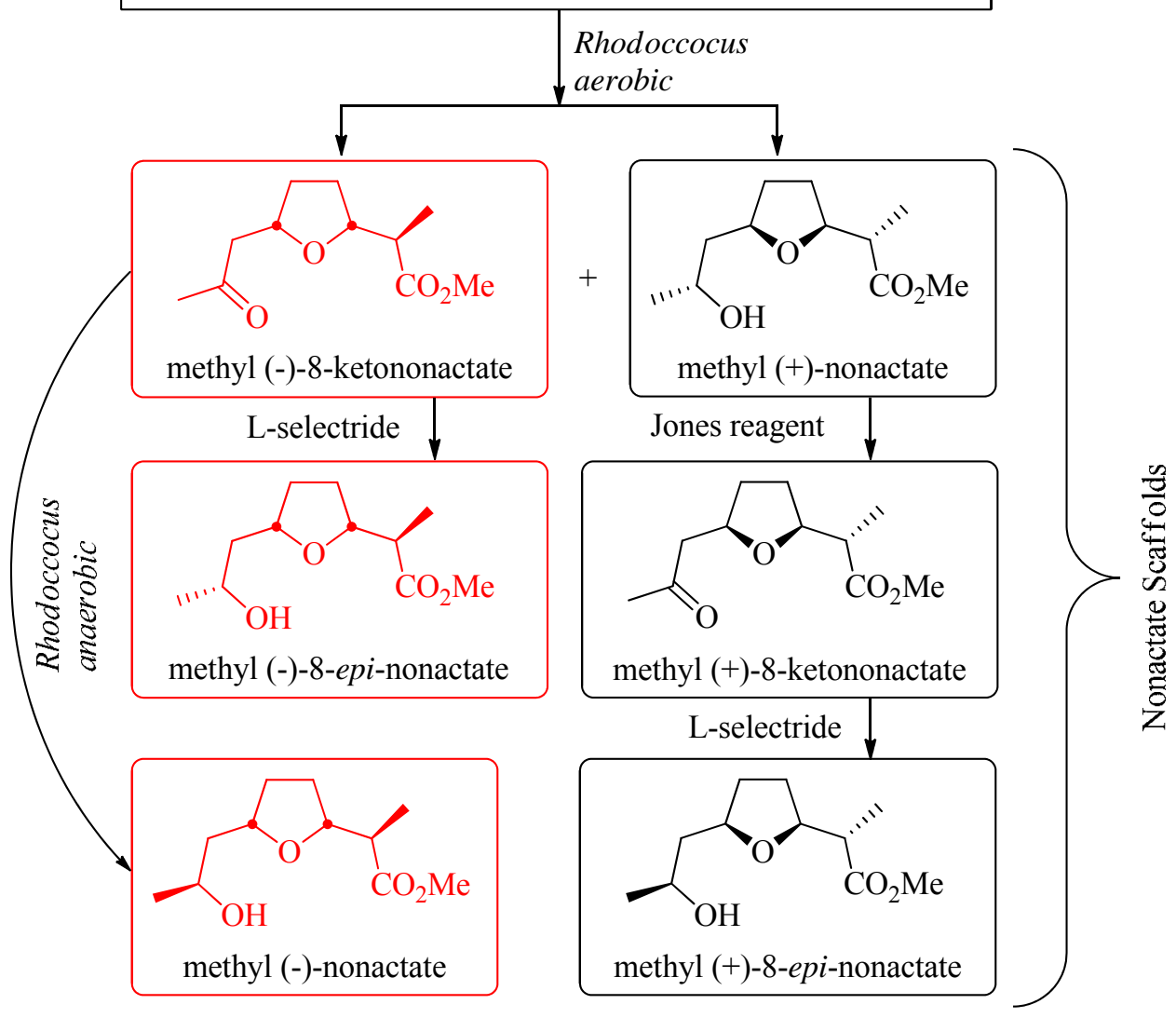

Scheme 1. Library building blocks from nonactin.

\section{Results and Discussion}

The design for a macrolide-like library from nonactate suggested an advanced scaffold that maintains several sites for diversification about the macrocycle while the embedded tetrahydrofuran unit imparts rigidity to the core structure, a beneficial effect seen with the third-generation 
erythromycin analogs (ketolides). ${ }^{7}$ We have termed this new class of natural-synthetic hybrids the nonactolides (Scheme 2).

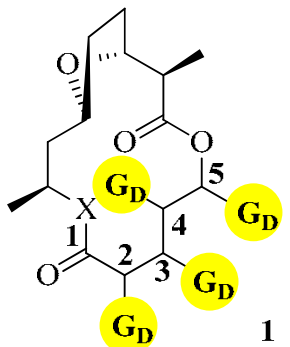

Macrolide-like Library

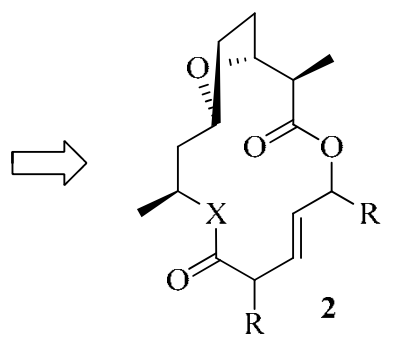

2
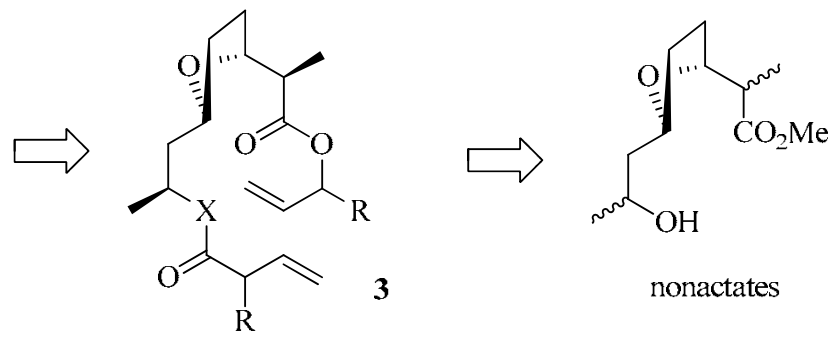

Scheme 2. Design of "nonactolide" libraries.

The long-term objective of this work is the preparation of a nonactolide library represented by $\mathbf{1}$ that builds upon the nonactate core. The precursor to this library would be a diversable scaffold such as 2 that retains several functional handles for diversity-oriented synthesis along the C2-C5 region such as a pendant alcohol, olefin and activated methylene group. It was envisioned that scaffolds of these types would arise by RCM reaction of 3, derived from functionalization of a nonactate building block. Initial efforts were based on a simple evaluation of the facility of RCM reaction of two olefins assembled on the nonactate core. Specifically, it was important to establish how the role of stereochemistry and substitution near the olefinic termini would impact the facility of the macrocyclic RCM reaction. Direct coupling of racemic nonactate to racemic fragments would produce a several diastereomers that could simply be studied as mixtures to evaluate the overall strategy. The precursors for the RCM reaction could be conveniently prepared by sequential coupling reactions (Scheme 3).

O-tert-Butyldimethylsilylnonactic acid 5 was prepared in two steps from racemic methyl nonactate 4 according to literature procedures. ${ }^{8}$ Carboxylic acid 5 was then esterified with either allyl alcohol or racemic 3-buten-2-ol to give esters $\mathbf{6 a}$ and $\mathbf{6 b}$, respectively as a mixture of diastereomers. Deprotection of the silyl ethers 6a/b was achieved upon exposure to a $70 \%$ solution of hydrofluoric acid in pyridine. Use of methoxytrimethylsilane as a non-aqueous quenching reagent provided alcohols $\mathbf{7 a} / \mathbf{b}$ that could be used in the next step without the benefit of further purification. Installing a second terminal alkene moiety via esterification of secondary alcohols 7a/b with either vinylacetic acid or 2-methyl-3-butenoic acid ${ }^{9}$ proved difficult. Initially, Mukaiyama resin ${ }^{10}$ was used to achieve this transformation. Unfortunately, the yields of the desired diesters 8ad were poor $(<30 \%)$, prompting a search for a more efficient esterification method. While DCC worked relatively well to convert 7a into $\mathbf{8 a}$, all attempts to use it or related carbodiimide reagents in the synthesis of $\mathbf{8 b}-\mathbf{d}$ were futile. Conversion of the carboxylic acids to the corresponding acid chlorides $^{11}$ ultimately allowed for successful acylation. Despite the formation of a large number of byproducts (possibly resulting from acid chloride conversion to ketene), diesters $\mathbf{8 b}$-d could be obtained in reasonable yields. 
<smiles>CC(=O)C(C)[C@H]1CC[C@@H](CCC(C)O)O1</smiles>

4
1) $\mathrm{TBSCl}$, imidazole, DMF

2) $\mathrm{KOH}, \mathrm{THF} / \mathrm{MeOH}$

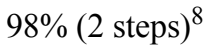

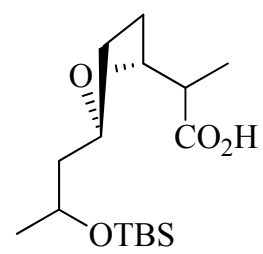

5

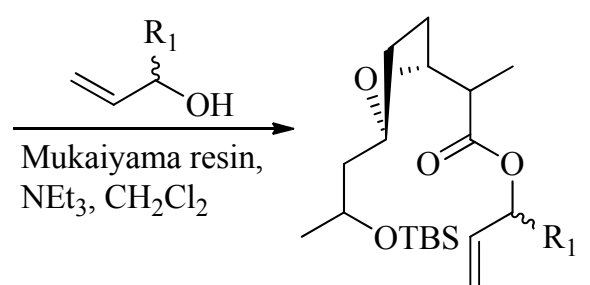

6a: $\mathrm{R}_{1}=\mathrm{H}, \quad 66-89 \%$ 6b: $\mathrm{R}_{1}=\mathrm{CH}_{3}, 60-86 \%$

HF/Py, THF then TMSOMe

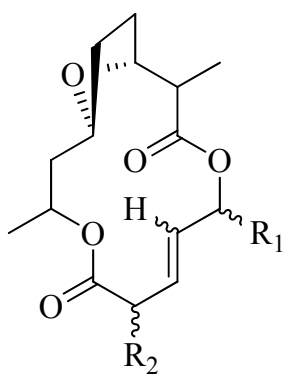

Grubbs' 2nd gen. cat. or Grubbs'-Hoveyda 2nd gen. cat.

\section{$\mathrm{CH}_{2} \mathrm{Cl}_{2}$} cis:trans ratio $1: 5$

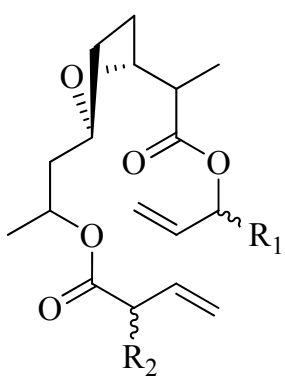

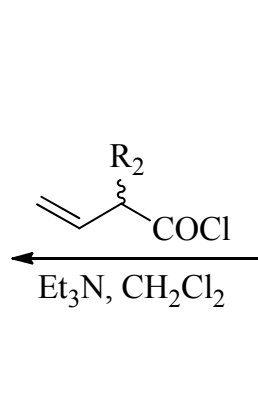

8a: $R_{1}=H, R_{2}=H$, 8b: $\mathrm{R}_{1}=\mathrm{H}, \mathrm{R}_{2}=\mathrm{CH}_{3}$, 8c: $\mathrm{R}_{1}=\mathrm{CH}_{3}, \mathrm{R}_{2}=\mathrm{H}$,

9a: $\mathrm{R}_{1}=\mathrm{H}, \mathrm{R}_{2}=\mathrm{H}$,

$81 \%$

9b: $\mathrm{R}_{1}=\mathrm{H}, \mathrm{R}_{2}=\mathrm{CH}_{3}, \quad 82 \%$

9c: $\mathrm{R}_{1}=\mathrm{CH}_{3}, \mathrm{R}_{2}=\mathrm{H}, \quad 82 \%$

9d: $\mathrm{R}_{1}=\mathrm{CH}_{3}, \mathrm{R}_{2}=\mathrm{CH}_{3}, 60 \%$

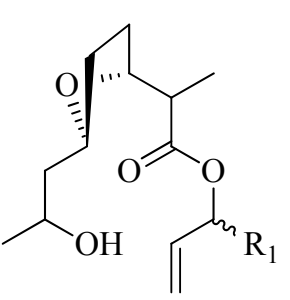

7a: $\mathrm{R}_{1}=\mathrm{H}, \quad 86 \%$

7b: $\mathrm{R}_{1}=\mathrm{CH}_{3}, 70 \%$

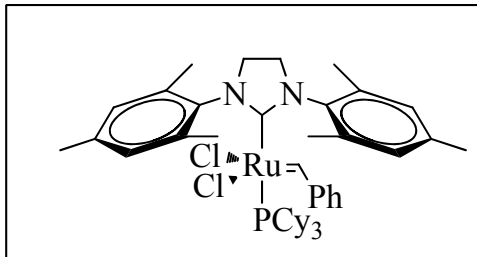

Grubbs' 2nd generation catalyst

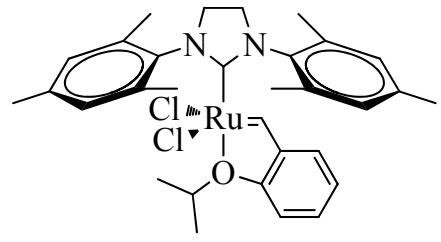

Grubbs'-Hoveyda 2nd generation catalyst

\section{Scheme 3}

With the four RCM precursors in hand (each as a mixture of diastereomers), we set out to determine optimal conditions for the key ring-closing olefin metathesis reaction. Intramolecular RCM reaction of 8a was initially carried out at three different concentrations $(10,5$ and $2.5 \mathrm{mM})$. Pleasingly, the desired product 9a was isolated as a 1:5 mixture of cis:trans isomers and in $81 \%$ yield at $2.5 \mathrm{mM}$. Running the reaction at the higher concentrations produced a dramatic diminution in yield an as such, all future RCM experiments were run only at $2.5 \mathrm{mM}$. From the distribution of products, it appeared that all of the diastereomers in the mixture cyclized with similar facility. Diesters $\mathbf{8 b}$ and $\mathbf{8 c}$ behaved similarly to $\mathbf{8 a}$ and were cyclized into the corresponding macrocycles 9b and 9c in similar yields. However, subjecting $\mathbf{8 d}$ to the same set of conditions resulted only in extensive decomposition of the starting material. By switching to Grubbs'-Hoveyda 2nd generation 
catalyst, increasing the catalyst load to $20 \mathrm{~mol} \%$ and increasing the reaction time to 5 days, we were able to isolate macrocycle 9d, albeit in lower yield

The result of these initial model cyclizations revealed that these 14-membered macrocycles could be formed directly by metathesis of an acyclic diene in reasonable yield and that the cyclization would be most effective with one pendant group in an allylic position. With the success of this initial model series, we set out to prepare more functionalized scaffolds as single enantiomers to be utilized in the generation of macrolide-like libraries. Of the two allylic positions that are installed on the nonactin scaffold we elected to introduce a diversable position at C5 since we envision that the carbonyl functionality at $\mathrm{C} 1$ will allow introduction of $\mathrm{C} 2$ diversity after formation of the nonactolide. This pendant functionality creates an additional stereogenic center in the scaffold and requires the preparation of the fragment in non-racemic form. We were attracted to the method developed by Trost ${ }^{12}$ for the asymmetric opening of vinyl oxiranes (Scheme 4).
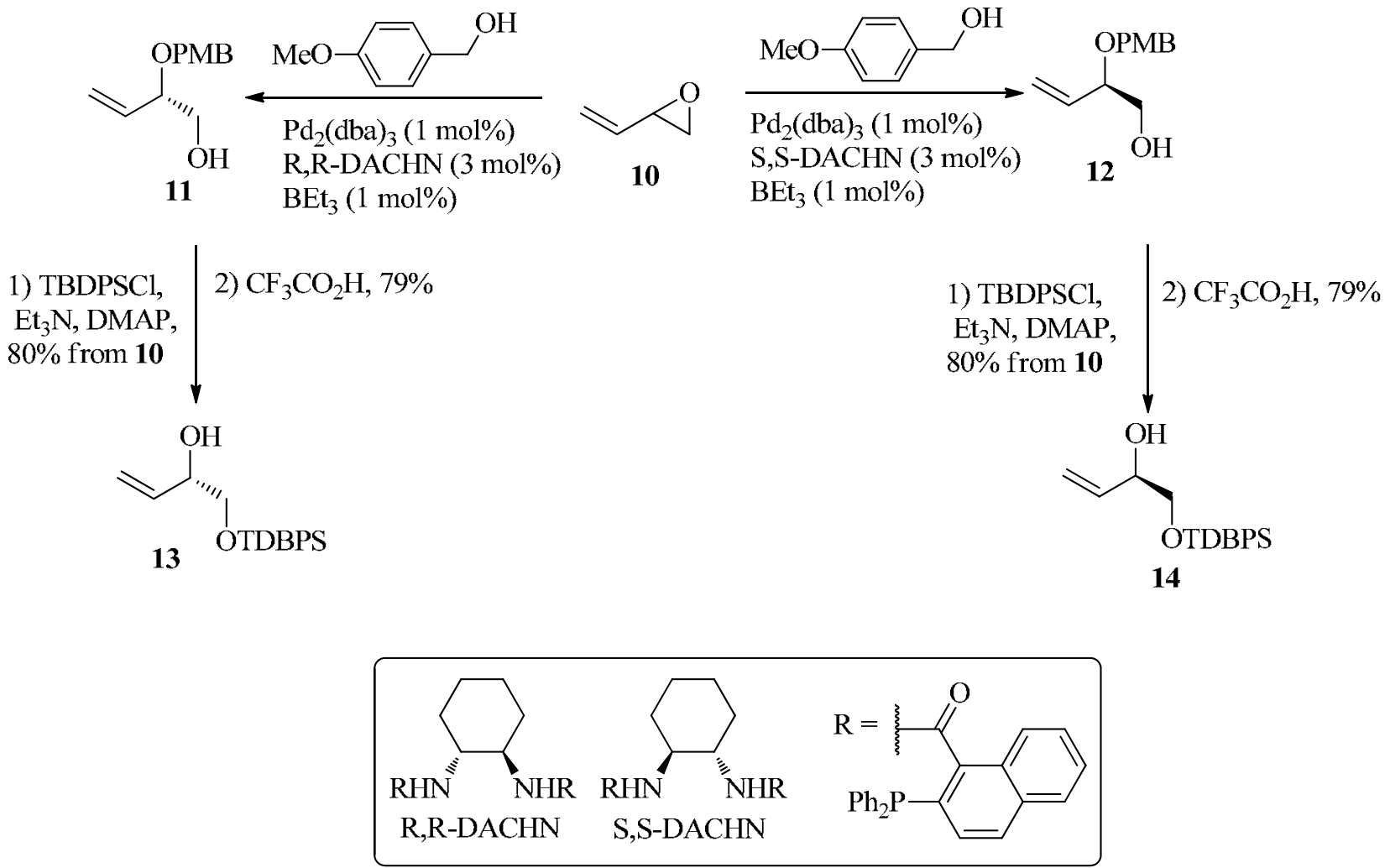

\section{Scheme 4}

Commercially available butadiene monoxide $\mathbf{1 0}$ was opened with $p$-methoxybenzyl alcohol in the presence of a palladium source and either R,R- or S,S-DACHN ligands to give the enantiomeric alcohols 11 and 12. Separation of the alcohols from residual $p$-methoxybenzyl alcohol proved difficult so the crude mixtures were converted to the corresponding OTBDMS ethers under standard conditions. After purification, desilylation could be effected to regenerate the pure alcohols and the specific optical rotations of compounds 11 and 12 were determined to be $[\alpha]_{\mathrm{D}}-43.1 \quad(\mathrm{c}=1.150$, $\left.\mathrm{CHCl}_{3}\right)$ and $[\alpha]_{\mathrm{D}}+46.8\left(\mathrm{c}=1.155, \mathrm{CHCl}_{3}\right)$, respectively. This was in good agreement with data 
reported for 11 by Trost $^{12}$ : $\left([\alpha]_{\mathrm{D}}-44.5,\left(\mathrm{c}=1.14, \mathrm{CHCl}_{3}\right), 91 \%\right.$ ee). The deblocked secondary alcohols 13 and 14 were best obtained by deprotection of the PMB groups with trifluoroacetic acid as the typical DDQ deprotection was problematic due to an inability to separate the product from $p$ methoxybenzaldehyde. With the enantiomerically enriched allylic alcohols in hand, elaboration of a non-racemic nonactate fragment was expected to follow from prior model studies (Scheme 5).

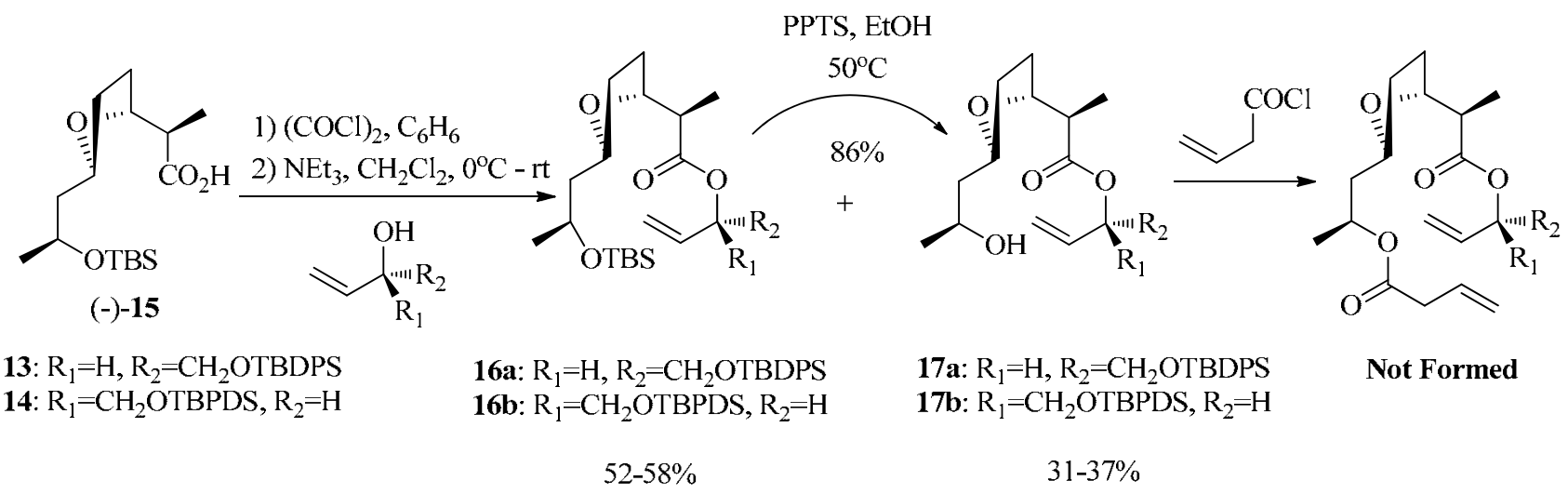

\section{Scheme 5}

Surprisingly, the secondary alcohols 13 and 14 turned out to be less reactive than the allylic alcohols used in the prior studies and could not be coupled under the previously developed conditions. Converting carboxylic acid (-)-15 into the acid chloride was necessary to facilitate ester formation to give $\mathbf{1 6 a} / \mathbf{b}$ which was accompanied by partial deprotection of the OTBS group. Heating an ethanolic solution of the reaction products with pyridinium $p$-toluenesulfonate completed removal of the OTBS group to give $\mathbf{1 7 a} / \mathbf{b}$. Unexpectedly, acylation of the secondary alcohol of $\mathbf{1 7} \mathbf{a} / \mathbf{b}$ with either vinyl acetyl chloride or vinyl acetic acid and a variety of coupling agents proved unsuccessful. Since the secondary hydroxyl group and the allylic substituent are separated by 10 atoms in both 7 and 17, a correlation between the size of allylic substituents and the reactivity of the secondary hydroxyl group was unanticipated. One possible explanation for this phenomenon is based on intramolecular hydrogen bond formation between the hydroxyl group and the oxygen atom of the tetrahydrofuran moiety. This interaction could lead to certain conformational changes that might bring the allylic side arm closer to the C8 hydroxyl group (nonactate numbering). In this case, the larger allylic substituents of alcohols 17a/b may shield the C8 hydroxyl, resulting in lower than expected reactivity. Because of this unexpected reactivity, the scaffold was revised to incorporate a C8 nitrogen in place of the oxygen atom to increase the nucleophilicity and hopefully result in productive acylation chemistry (Scheme 6). 


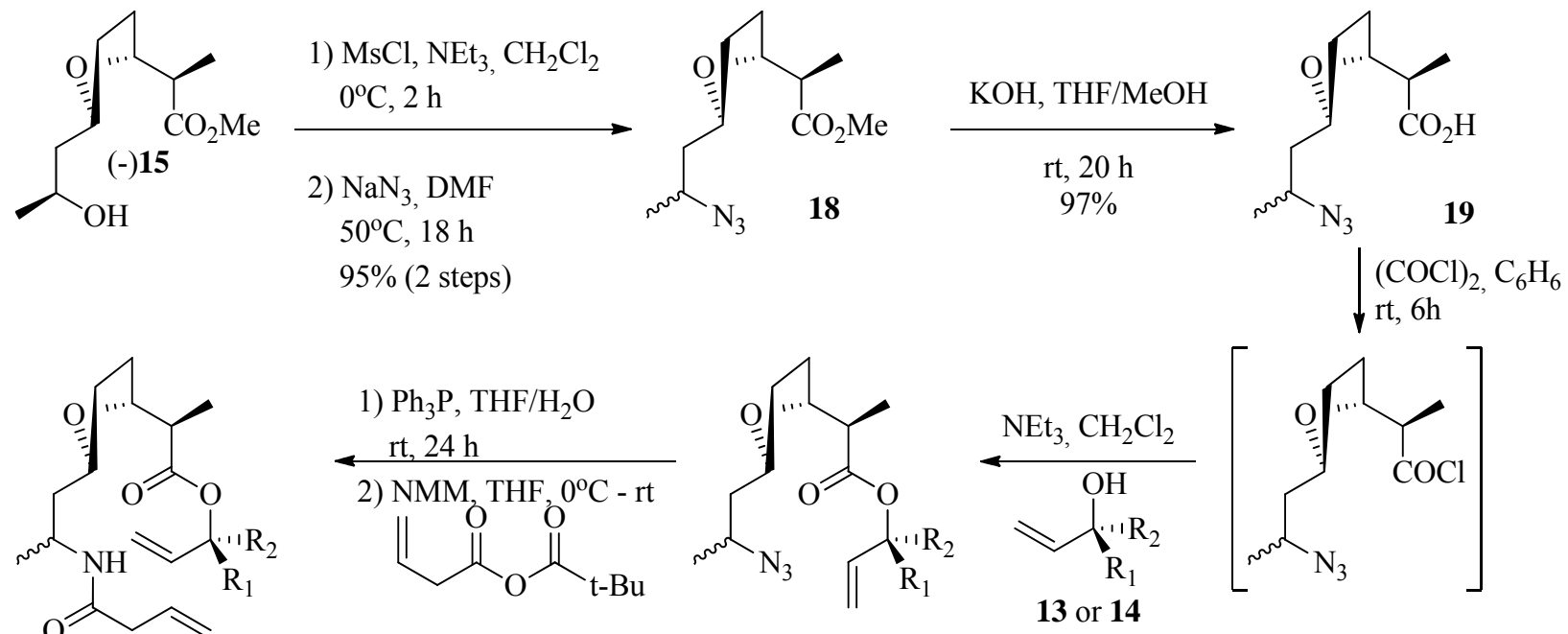

21a: $\mathrm{R}_{1}=\mathrm{H}, \mathrm{R}_{2}=\mathrm{CH}_{2}$ OTBDPS $82 \%$

21b: $\mathrm{R}_{1}=\mathrm{CH}_{2} \mathrm{OTBPDS}, \mathrm{R}_{2}=\mathrm{H} \quad 64 \%$

20a: $\mathrm{R}_{1}=\mathrm{H}, \mathrm{R}_{2}=\mathrm{CH}_{2}$ OTBDPS $88 \%$

20b: $\mathrm{R}_{1}=\mathrm{CH}_{2} \mathrm{OTBPDS}, \mathrm{R}_{2}=\mathrm{H} \quad 91 \%$

\section{Scheme 6}

Methyl nonactate (-)15 was converted to the corresponding azide through the intermediacy of the mesylate. Surprisingly, under these fairly standard conditions, racemization of the C8stereogenic center was observed, leading to an inseparable mixture of azides $\mathbf{1 8}$. We elected to continue on with this mixture to fully evaluate the design and hopefully separate the diastereomers at a later point in the synthesis. Alkaline hydrolysis of $\mathbf{1 8}$ afforded carboxylic acids $\mathbf{1 9}$ in nearly quantitative yield, which were elaborated to esters 20a/b as previously described via an acid chloride intermediate. Reduction of the azide moiety with triphenylphosphine occurred smoothly and pleasingly the crude amines could converted into amides 21a and 21b by mixed anhydride acylation. ${ }^{13}$ as a prelude to the key RCM reaction (Scheme 7).

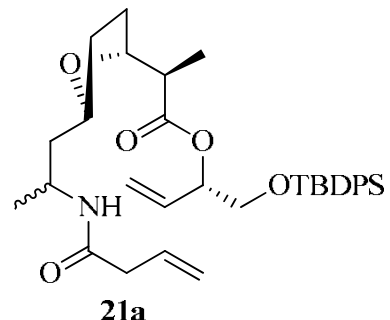

21a

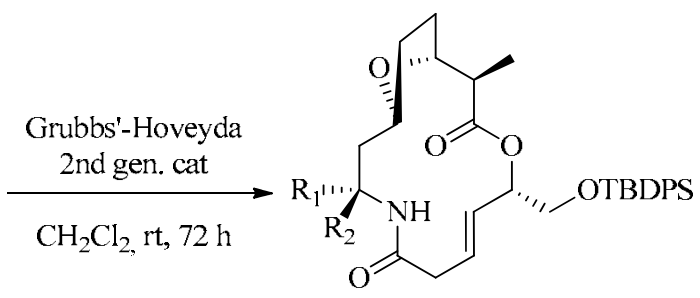

22a: $\mathrm{R}_{1}=\mathrm{H}, \mathrm{R}_{2}=\mathrm{CH}_{3} \quad 33 \%$

22b: $\mathrm{R}_{1}=\mathrm{CH}_{3}, \mathrm{R}_{2}=\mathrm{H} \quad 35 \%$

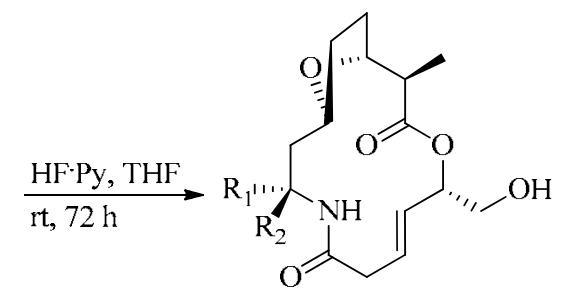

24a: $\mathrm{R}_{1}=\mathrm{H}, \mathrm{R}_{2}=\mathrm{CH}_{3} \quad 82 \%$ 24b: $\mathrm{R}_{1}=\mathrm{CH}_{3}, \mathrm{R}_{2}=\mathrm{H} \quad 76 \%$

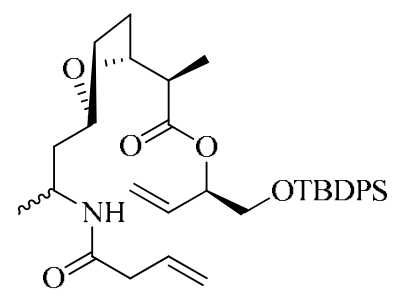

21b

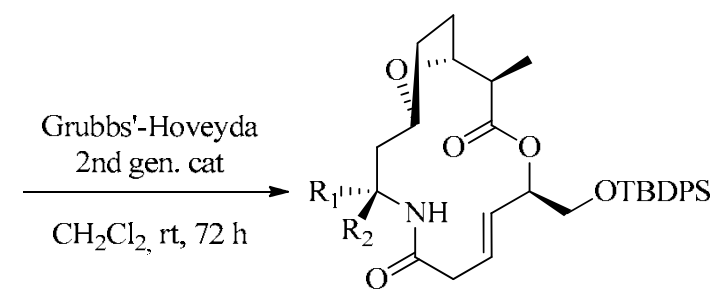

23a: $\mathrm{R}_{1}=\mathrm{H}, \mathrm{R}_{2}=\mathrm{CH}_{3} \quad 50 \%$ 23b: $\mathrm{R}_{1}=\mathrm{CH}_{3}, \mathrm{R}_{2}=\mathrm{H} \quad 32 \%$

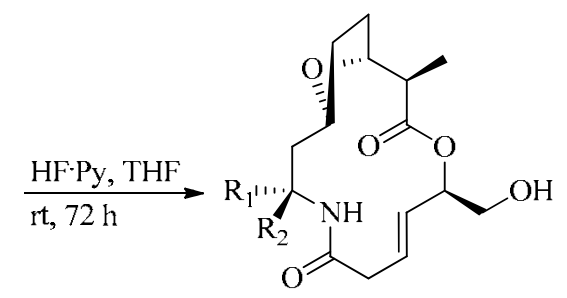

25a: $\mathrm{R}_{1}=\mathrm{H}, \mathrm{R}_{2}=\mathrm{CH}_{3} \quad 79 \%$ 25b: $\mathrm{R}_{1}=\mathrm{CH}_{3}, \mathrm{R}_{2}=\mathrm{H} \quad 80 \%$

\section{Scheme 7}


Although reaction times and catalyst loading had to be increased compared to the conditions used to cyclize diesters 8a-d, we were able to isolate 14-membered macrocyclic compounds 22a/b and $\mathbf{2 3 a} / \mathbf{b}$, in reasonable yields. In addition, it was gratifying to find that we could affect separation of the two C8-epimers by simple chromatography. The identity of each isomer was assigned by NMR analysis of NOESY correlations between the $\alpha$-amino proton and protons on the tetrahydorfuran ring and allylic protons in the macrocycle. As before, the trans isomer predominates ( $>5: 1$ ratio by NMR analysis of the crude mixture) with the minor cis isomer being simply removed by chromatography. Final deprotection of tert-butyldiphenylsilyl ethers was achieved by using a 70 $\%$ solution of hydrofluoric acid in pyridine to produce the scaffolds 24a, 24b, 25a and 25b. These four enantiomerically pure scaffolds contain up to four sites for diversification including the activate methylene, alkene and the pendant primary alcohol.

\section{Conclusions}

As part of a long term program aimed at the development of natural product-like libraries derived from natural feedstocks, we have focused on the development of high complexity scaffolds to be used in library synthesis. In this manuscript, we report on studies aimed at the development of macrolide-like scaffolds derived form various isomers of nonactate. The resultant "nonactolides" will represent a scalable and economical collection of diversable scaffolds rich in stereochemical as well as substituent diversity. Use of a highly effective RCM reaction on a nonactate-derived diene delivered the scaffolds with up to 4 positions that can be used to introduce diversity into the structure. The effectiveness of RCM reactions was essentially independent of the configuration of the stereogenic centers present in diene but was sensitive to the presence of substituents in the allylic position. Current efforts are focused on the diversification of the nonactolide scaffolds to produce natural product-like libraries.

\section{Experimental Section}

( \pm )-O-(tert-Butyldimethylsilyl)nonactic acid allyl ester (6a). To a solution of $1.56 \mathrm{~g} \mathrm{(4.9} \mathrm{mmol,}$ $1.0 \mathrm{eq})$ of carboxylic acid 5 in $5 \mathrm{~mL}$ of $\mathrm{CH}_{2} \mathrm{Cl}_{2}$ were added $2.13 \mathrm{~g}$ (10.3 mmol, $\left.2.1 \mathrm{eq}\right)$ of 1,3dicyclohexylcarbodiimide, $0.44 \mathrm{~mL}(6.4 \mathrm{mmol}, 1.3 \mathrm{eq})$ of allyl alcohol and a catalytic amount of $4-$ (dimethylamino)-pyridine. The resulting mixture was stirred at room temperature for $16 \mathrm{~h}$. After evaporation of the solvent under reduced pressure the crude product was purified by flash chromatography $\left(\mathrm{SiO}_{2}, 87 \mathrm{~g}, 1 \%\right.$ ether/pentane $\rightarrow 2 \%$ ether/pentane) giving ester $6 \mathbf{a}$ as a colorless oil (1.31 g, 74\%): TLC $\mathrm{R}_{\mathrm{f}}=0.33$ (5\% ether/pentane); IR (neat, $\mathrm{KBr}, \mathrm{cm}^{-1}$ ) 2957, 2930, 2883, 2856, 1740, 1462, 1377, 1256, 1153, 1070, 987, 837, 775; ${ }^{1} \mathrm{H}$ NMR (500 MHz, $\left.\mathrm{CDCl}_{3}\right) \delta 5.92(\mathrm{ddt}, J=$ $17.1,10.5,5.6 \mathrm{~Hz}, 1 \mathrm{H}), 5.32(\mathrm{ddd}, J=17.1,3.0,1.5 \mathrm{~Hz}, 1 \mathrm{H}), 5.21$ (ddd, $J=10.5,3.0,1.5 \mathrm{~Hz}, 1 \mathrm{H})$, $4.62(\mathrm{ddt}, J=13.4,5.6,1.4 \mathrm{~Hz}, 1 \mathrm{H}), 4.56(\mathrm{ddt}, J=13.4,5.6,1.4 \mathrm{~Hz}, 1 \mathrm{H}), 3.99-3.86(\mathrm{~m}, 3 \mathrm{H}), 2.52$ $(\mathrm{dq}, J=14.1,7.0 \mathrm{~Hz}, 1 \mathrm{H}), 2.00-1.89(\mathrm{~m}, 2 \mathrm{H}), 1.61-1.52(\mathrm{~m}, 3 \mathrm{H}), 1.52-1.41(\mathrm{~m}, 1 \mathrm{H}), 1.12(\mathrm{~d}, J$ 
$5.3 \mathrm{~Hz}, 3 \mathrm{H}), 1.11(\mathrm{~d}, J=4.4 \mathrm{~Hz}, 3 \mathrm{H}), 0.87$ (bs, 9H), 0.04 (s, 3H), 0.03 (s, 3H); ${ }^{13} \mathrm{C} \mathrm{NMR}(125 \mathrm{MHz}$, $\left.\mathrm{CDCl}_{3}\right) \delta 174.7,132.5,118.0,80.4,76.4,66.3,65.1,46.2,45.8,31.5,28.9,26.1,24.8,18.2,13.6$, 4.4, -4.7; ESI-HRMS m/z calcd for $\mathrm{C}_{19} \mathrm{H}_{37} \mathrm{O}_{4} \mathrm{Si}[\mathrm{M}+\mathrm{H}]^{+}$357.2456, found 357.2447; ESI-HRMS $\mathrm{m} / \mathrm{z}$ calcd for $\mathrm{C}_{19} \mathrm{H}_{36} \mathrm{NaO}_{4} \mathrm{Si}[\mathrm{M}+\mathrm{Na}]^{+}$379.2275, found 379.2265.

( \pm )-O-(tert-Butyldimethylsilyl)nonactic acid 3-buten-2-olyl ester (6b). To a solution of $0.0624 \mathrm{~g}$ $(0.20 \mathrm{mmol}, 1.0 \mathrm{eq})$ of carboxylic acid 5 in $2 \mathrm{~mL}$ of $\mathrm{CH}_{2} \mathrm{Cl}_{2}$ were added $0.093 \mathrm{~g}(0.49 \mathrm{mmol}, 2.5$ eq) of $\mathrm{EDC} \cdot \mathrm{HCl}$, and $0.101 \mathrm{~g}(0.83 \mathrm{mmol}, 4.2 \mathrm{eq})$ of 4 -(dimethylamino)pyridine. The resulting mixture was stirred at room temperature for $1 \mathrm{~h}$. Then, $44 \mathrm{~mL}(0.49 \mathrm{mmol}, 2.5 \mathrm{eq})$ of 3-buten-2-ol was added, and the reaction mixture was stirred at room temperature for another $16 \mathrm{~h}$. After evaporation of the solvent under reduced pressure, $3 \mathrm{~mL}$ of ether was added to the crude product, followed by $1 \mathrm{~mL}$ of ice-cold $\mathrm{HCl}(1.0 \mathrm{M})$. The resulting emulsion was shaken vigorously, organic layer was separated and the aqueous layer was extracted with ether $(3 * 2 \mathrm{~mL})$. The combined organic extracts were dried over $\mathrm{MgSO}_{4}$ and concentrated under reduced pressure. Flash chromatography $\left(\mathrm{SiO}_{2}, 8 \mathrm{~g}, 1 \%\right.$ ether/hexanes $\rightarrow 2 \%$ ether/hexanes) afforded $0.0314 \mathrm{~g}(43 \%)$ of ester $\mathbf{6 b}$ as a colorless oil (mixture of diastereomers): TLC $\mathrm{R}_{\mathrm{f}}=0.37$ (10\% ether/hexanes); IR (neat, $\mathrm{KBr}, \mathrm{cm}^{-1}$ ) 2955, 2928, 2856, 1801, 1742, 1462, 1375, 1256, 1068, 837, 775; ${ }^{1} \mathrm{H} \mathrm{NMR}\left(500 \mathrm{MHz}, \mathrm{CDCl}_{3}\right) \delta$ (mixture) $5.89-5.80(\mathrm{~m}, 1 \mathrm{H}), 5.37(\mathrm{p}, J=6.3 \mathrm{~Hz}, 1 \mathrm{H}), 5.29-5.22(\mathrm{~m}, 1 \mathrm{H}), 5.14-5.09(\mathrm{~m}, 1 \mathrm{H})$, $4.03-3.85(\mathrm{~m}, 3 \mathrm{H}), 2.49$ (p, J= $7.1 \mathrm{~Hz}, 1 \mathrm{H}), 2.01-1.87(\mathrm{~m}, 2 \mathrm{H}), 1.62-1.52(\mathrm{~m}, 2 \mathrm{H}), 1.52-1.39$ $(\mathrm{m}, 2 \mathrm{H}), 1.31(\mathrm{dd}, J=6.5,1.8 \mathrm{~Hz}, 3 \mathrm{H}), 1.13-1.08(\mathrm{~m}, 6 \mathrm{H}), 0.87(\mathrm{~s}, 9 \mathrm{H}), 0.04(\mathrm{~s}, 6 \mathrm{H}) ;{ }^{13} \mathrm{C}$ NMR $\left(125 \mathrm{MHz}, \mathrm{CDCl}_{3}\right) \delta$ (mixture) 174.3, 174.2, 138.0, 115.7, 115.5, 80.4, 80.3, 76.4, 70.8, 70.7, 66.4, 66.3, 46.3, 46.2, 45.9, 45.8, 31.6, 28.7, 26.1, 24.8, 20.1, 20.0, 18.2, 13.5, 13.4, -4.4, -4.7; ESIHRMS $m / z$ calcd for $\mathrm{C}_{20} \mathrm{H}_{39} \mathrm{O}_{4} \mathrm{Si}[\mathrm{M}+\mathrm{H}]^{+}$371.2612, found 371.2610; ESI-HRMS m/z calcd for $\mathrm{C}_{20} \mathrm{H}_{38} \mathrm{NaO}_{4} \mathrm{Si}[\mathrm{M}+\mathrm{Na}]^{+}$393.2432, found 393.2435.

\section{General procedure for TBS deprotection}

To a $0.1 \mathrm{M}$ solution of either TBS protected alcohol $\mathbf{6 a}$ or $\mathbf{6 b}$ (1.0 eq) in THF was added a solution of HF in pyridine (20.0 eq, $70 \%$ by weight) in one portion. The resulting solution was stirred at room temperature for $18 \mathrm{~h}$ until TLC showed no starting material. Methoxytrimethylsilane (60.0 eq) was added and the reaction mixture was stirred vigorously for $10 \mathrm{~min}$ to neutralize the excess of HF. After evaporation of the solvent under reduced pressure the crude product was purified by flash chromatography $\left(\mathrm{SiO}_{2}, 1: 50\right.$ ratio of crude product to silica gel).

$( \pm)$-Nonactic acid allyl ester (7a). Using the general procedure as described above, TBS-protected alcohol 6a $(0.083 \mathrm{~g}, 0.23 \mathrm{mmol})$ was reacted to give alcohol 48a $(0.048 \mathrm{~g}, 86 \%)$ as a colorless oil after flash chromatography $\left(\mathrm{SiO}_{2}, 3 \mathrm{~g}, 20 \%\right.$ ether/pentane $\rightarrow 25 \%$ ether/pentane): TLC $\mathrm{R}_{\mathrm{f}}=0.21$ (40\% ether/pentane); IR (neat, $\mathrm{KBr}, \mathrm{cm}^{-1}$ ) 3429 (broad), 2968, 2937, 2360, 2341, 1738, 1462, 1456, 1377, 1271, 1250, 1186, 1159, 1090, 1059, 935; ${ }^{1} \mathrm{H}$ NMR (500 MHz, $\left.\mathrm{CDCl}_{3}\right) \delta 5.91(\mathrm{ddt}, J=17.2$, 10.5, $5.7 \mathrm{~Hz}, 1 \mathrm{H}), 5.31$ (ddd, $J=17.2,3.0 \mathrm{~Hz}, 1.4 \mathrm{~Hz}, 1 \mathrm{H}), 5.21$ (ddd, $J=10.5,3.0,1.4 \mathrm{~Hz}, 1 \mathrm{H})$, 4.59 (dt, $J 5.7,1.4 \mathrm{~Hz}, 2 \mathrm{H}), 4.12(\mathrm{qd}, J=7.3,4.0 \mathrm{~Hz}, 1 \mathrm{H}), 4.05-3.96(\mathrm{~m}, 2 \mathrm{H}), 2.55(\mathrm{dq}, J=8.1$, $7.0 \mathrm{~Hz}, 1 \mathrm{H}), 2.51$ (bs, 1H), $2.05-1.92(\mathrm{~m}, 2 \mathrm{H}), 1.72(\mathrm{ddd}, J=14.3,7.8,4.0 \mathrm{~Hz}, 1 \mathrm{H}), 1.67-1.56$ $(\mathrm{m}, 3 \mathrm{H}), 1.19(\mathrm{~d}, J=6.3 \mathrm{~Hz}, 3 \mathrm{H}), 1.13(\mathrm{~d}, J=7.0 \mathrm{~Hz}, 3 \mathrm{H}) ;{ }^{13} \mathrm{C}$ NMR $\left(125 \mathrm{MHz}, \mathrm{CDCl}_{3}\right) \delta 174.6$, $132.3,118.3,81.1,77.3,65.3,65.2,45.5,43.0,30.7,28.9,23.4,13.7$; ESI-HRMS $\mathrm{m} / \mathrm{z}$ calcd for 
$\mathrm{C}_{13} \mathrm{H}_{23} \mathrm{O}_{4}[\mathrm{M}+\mathrm{H}]^{+}$243.1591, found 243.1597; ESI-HRMS m/z calcd for $\mathrm{C}_{13} \mathrm{H}_{22} \mathrm{NaO}_{4}[\mathrm{M}+\mathrm{Na}]^{+}$ 265.1410 , found 265.1416.

( \pm )-Nonactic acid 3-buten-2-olyl ester (7b). Using the general procedure as described above, TBSprotected alcohol $\mathbf{6 b}(0.021 \mathrm{~g}, 0.06 \mathrm{mmol})$ was reacted to give $0.010 \mathrm{~g}(70 \%)$ of alcohol $7 \mathbf{b}$ as a colorless oil (mixture of diastereomers) after flash chromatography $\left(\mathrm{SiO}_{2}, 1 \mathrm{~g}, 15 \%\right.$ ether/pentane $\rightarrow 20 \%$ ether/pentane): TLC $\mathrm{R}_{\mathrm{f}}=0.15$ (40\% ether/hexanes); IR (neat, $\mathrm{KBr}, \mathrm{cm}^{-1}$ ) 3447 (broad), 2964, 2928, 2874, 1734, 1458, 1375, 1259, 1190, 1167, 1138, 1088, 1049, 991, 935; ${ }^{\mathrm{H}} \mathrm{NMR}(500$ $\left.\mathrm{MHz}, \mathrm{CDCl}_{3}\right) \delta$ (mixture) 5.84 (dddd, $\left.J=17.3,10.5,5.8,1.4 \mathrm{~Hz}, 1 \mathrm{H}\right), 5.41-5.33(\mathrm{~m}, 1 \mathrm{H}), 5.29-$ $5.21(\mathrm{~m}, 1 \mathrm{H}), 5.13(\mathrm{ddd}, J=10.5,4.1,1.0 \mathrm{~Hz}, 1 \mathrm{H}), 4.18-4.08(\mathrm{~m}, 1 \mathrm{H}), 4.08-3.95(\mathrm{~m}, 2 \mathrm{H}), 2.59-$ $2.48(\mathrm{~m}, 1 \mathrm{H}), 2.20(\mathrm{bs}, 1 \mathrm{H}), 2.03-1.92(\mathrm{~m}, 2 \mathrm{H}), 1.77-1.54(\mathrm{~m}, 4 \mathrm{H}), 1.31(\mathrm{dd}, J=6.5,4.5 \mathrm{~Hz}$, $3 \mathrm{H}), 1.20(\mathrm{dd}, J=6.4,1.4 \mathrm{~Hz}, 3 \mathrm{H}), 1.12(\mathrm{dd}, J=7.0,2.5 \mathrm{~Hz}, 3 \mathrm{H}) ;{ }^{1} \mathrm{H}$ NMR $\left(500 \mathrm{MHz}, \mathrm{C}_{6} \mathrm{D}_{6}\right) \delta$ (mixture) 5.79 (dddd, $J=20.3,17.1,10.6,5.7 \mathrm{~Hz}, 1 \mathrm{H}), 5.54-5.44(\mathrm{~m}, 1 \mathrm{H}), 5.19$ (ddt, $J=22.4$, $17.3,1.4 \mathrm{~Hz}, 1 \mathrm{H}), 4.98(\mathrm{ddt}, J=22.7,10.6,1.3 \mathrm{~Hz}, 1 \mathrm{H}), 4.04-3.83(\mathrm{~m}, 3 \mathrm{H}), 2.46(\mathrm{ddq}, J=14.0$, 11.2, 7.0 Hz, 1H), $2.40(\mathrm{~s}, 1 \mathrm{H}), 1.57-1.42(\mathrm{~m}, 4 \mathrm{H}), 1.42-1.26(\mathrm{~m}, 2 \mathrm{H}), 1.21(\mathrm{dd}, J=31.7,6.5 \mathrm{~Hz}$, $3 \mathrm{H}), 1.14(\mathrm{dd}, J=6.3,3.0 \mathrm{~Hz}, 3 \mathrm{H}), 0.99(\mathrm{dd}, J=10.6,7.0 \mathrm{~Hz}, 3 \mathrm{H}) ;{ }^{13} \mathrm{C} \mathrm{NMR}\left(125 \mathrm{MHz}, \mathrm{CDCl}_{3}\right) \delta$ (mixture) 174.2, 174.1, 137.9, 137.8, 116.0, 115.8, 81.2, 81.1, 77.3, 77.2, 71.2, 71.1, 65.4, 45.6, 43.1, 42.9, 30.8, 30.7, 28.9, 28.8, 23.4, 23.3, 20.1, 20.0, 13.6, 13.5; ${ }^{13} \mathrm{C}$ NMR $\left(125 \mathrm{MHz}, \mathrm{C}_{6} \mathrm{D}_{6}\right) \delta$ (mixture) 173.9, 173.8, 138.7, 138.6, 115.9, 115.8, 81.6, 81.4, 77.6, 77.5, 71.3, 71.2, 65.4, 46.1, 44.2, 44.0, 31.3, 31.2, 29.1, 29.0, 24.2, 24.1, 20.4, 20.3, 13.9, 13.8; ${ }^{13} \mathrm{C} \mathrm{NMR}\left(125 \mathrm{MHz}, \mathrm{C}_{6} \mathrm{D}_{6}\right) \delta$ 173.6, 173.5, 138.4, 138.3, 115.6, 115.5, 81.3, 81.1, 77.2, 77.1, 71.0, 70.9, 65.1, 45.8, 45.7, 43.8, 43.7, 31.0, 30.9, 28.8, 28.7, 23.9, 23.8, 20.1, 20.0, 13.6, 13.5; ESI-HRMS m/z calcd for $\mathrm{C}_{14} \mathrm{H}_{25} \mathrm{O}_{4}$ $[\mathrm{M}+\mathrm{H}]^{+}$257.1747, found 257.1756; ESI-HRMS $\mathrm{m} / \mathrm{z}$ calcd for $\mathrm{C}_{14} \mathrm{H}_{24} \mathrm{NaO}_{4}[\mathrm{M}+\mathrm{Na}]^{+} 279.1567$, found 279.1575 .

But-3-enoic acid 2-[5-(1-allyloxycarbonyl-ethyl)-tetrahydrofuran-2-yl]-1-methyl-ethyl ester (8a). A solution of $0.36 \mathrm{~mL}$ (4.11 mmol, $2.1 \mathrm{eq})$ of vinylacetic acid and $0.847 \mathrm{~g}(4.11 \mathrm{mmol}, 2.1 \mathrm{eq})$ of 1,3-dicyclohexylcarbodiimide in $16 \mathrm{~mL}$ of $\mathrm{CH}_{2} \mathrm{Cl}_{2}$ was stirred at room temperature for $30 \mathrm{~min}$. Then, a solution of $0.4741 \mathrm{~g}(1.96 \mathrm{mmol}, 1.0 \mathrm{eq})$ of alcohol 7a in $4 \mathrm{~mL}$ of $\mathrm{CH}_{2} \mathrm{Cl}_{2}$ was added, followed by a catalytic amount of of 4-(dimethylamino)pyridine. The resulting mixture was stirred at room temperature for $24 \mathrm{~h}$. After evaporation of the solvent under reduced pressure, the crude product was purified by flash chromatography $\left(\mathrm{SiO}_{2}, 60 \mathrm{~g}, 6 \%\right.$ ether/pentane $\rightarrow 8 \%$ ether/pentane) afforded diester 8a as a colorless oil $(0.411 \mathrm{~g}, 67 \%)$ : TLC $\mathrm{R}_{\mathrm{f}}=0.31$ (20\% ether/pentane); IR (neat, $\mathrm{KBr}, \mathrm{cm}^{-1}$ ) 2976, 2939, 2880, 1738, 1732, 1643, 1462, 1379, 1325, 1254, 1178, 1090, 1059, 993, 922; ${ }^{1} \mathrm{H}$ NMR (500 MHz, $\left.\mathrm{CDCl}_{3}\right) \delta 5.89-5.77(\mathrm{~m}, 2 \mathrm{H}), 5.25(\mathrm{ddd}, J=17.2,2.9,1.5 \mathrm{~Hz}, 1 \mathrm{H}), 5.14$ (ddd, $J=10.5,2.5,1.5 \mathrm{~Hz}, 1 \mathrm{H}), 5.10-5.06(\mathrm{~m}, 1 \mathrm{H}), 5.06-5.03(\mathrm{~m}, 1 \mathrm{H}), 4.95-4.86(\mathrm{~m}, 1 \mathrm{H}), 4.57$ $-4.46(\mathrm{~m}, 2 \mathrm{H}), 3.97-3.88(\mathrm{~m}, 1 \mathrm{H}), 3.85-3.76(\mathrm{~m}, 1 \mathrm{H}), 2.97(\mathrm{dt}, J=7.0,1.3 \mathrm{~Hz}, 2 \mathrm{H}), 2.44(\mathrm{dq}, J$ $=14.1,7.0 \mathrm{~Hz}, 1 \mathrm{H}), 1.95-1.83(\mathrm{~m}, 2 \mathrm{H}), 1.70(\mathrm{ddd}, J=13.0,8.0,4.9 \mathrm{~Hz}, 1 \mathrm{H}), 1.62(\mathrm{ddd}, J=12.9$, 8.9, $5.0 \mathrm{~Hz}, 1 \mathrm{H}), 1.58-1.49(\mathrm{~m}, 1 \mathrm{H}), 1.48-1.39(\mathrm{~m}, 1 \mathrm{H}), 1.15(\mathrm{~d}, J=6.3 \mathrm{~Hz}, 3 \mathrm{H}), 1.04(\mathrm{~d}, J=7.1$ $\mathrm{Hz}, 3 \mathrm{H}) ;{ }^{13} \mathrm{C}$ NMR $\left(125 \mathrm{MHz}, \mathrm{CDCl}_{3}\right) \delta 174.3,170.8,132.2,130.4,118.2,117.9,80.4,76.2,69.5$, 64.9, 45.4, 42.4, 39.4, 31.2, 28.4, 20.5, 13.3; ESI-HRMS m/z calcd for $\mathrm{C}_{17} \mathrm{H}_{27} \mathrm{O}_{5}[\mathrm{M}+\mathrm{H}]^{+}$ 311.1853, found 311.1859; ESI-HRMS $\mathrm{m} / \mathrm{z}$ calcd for $\mathrm{C}_{14} \mathrm{H}_{24} \mathrm{NaO}_{4}[\mathrm{M}+\mathrm{Na}]^{+} 333.1672$, found 333.1682 . 


\section{General esterification procedure using acid chlorides}

To a $0.2 \mathrm{M}$ solution of either alcohol 7a or alcohol $7 \mathbf{b}(1.0 \mathrm{eq})$ in anhydrous $\mathrm{CH}_{2} \mathrm{Cl}_{2}$ cooled to $0^{\circ} \mathrm{C}$ were added $\mathrm{N}$-methylmorpholine (3.0 eq), either vinylacetic acid chloride or 2-methyl-3-butenoic acid chloride (2.0 eq) and a catalytic amount of 4-(dimethylamino)pyridine. The resulting mixture was stirred at $0^{\circ} \mathrm{C}$ for $30 \mathrm{~min}$ and then at room temperature for 1-2 hr. After evaporation of the solvent under reduced pressure, the crude product was dissolved in an ice-cold 3:1 emulsion of ether and $1.0 \mathrm{M} \mathrm{HCl}(3.0 \mathrm{eq})$. The resulting emulsion was shaken vigorously, organic layer was separated and the aqueous layer was extracted with ether. The combined organic extracts were dried over $\mathrm{MgSO}_{4}$. After evaporation of the solvent under reduced pressure, the crude product was purified by flash chromatography $\left(\mathrm{SiO}_{2}, 1: 100\right.$ ratio of crude product to silica gel).

2-Methyl-but-3-enoic acid 2-[5-(1-allyloxycarbonylethyl)tetrahydrofuran-2-yl]-1-methyl-ethyl ester (8b). Using the general procedure as described above, alcohol 7a $(0.119 \mathrm{~g}, 0.49 \mathrm{mmol})$ was reacted to give $0.142 \mathrm{~g}(88 \%)$ of the diester $\mathbf{8 b}$ as a colorless oil (mixture of diastereomers) after flash chromatography ( $\mathrm{SiO}_{2}, 25 \mathrm{~g}, 5 \%$ ether/hexanes $\rightarrow 7 \%$ ether/hexanes): TLC $\mathrm{R}_{\mathrm{f}}=0.31(20 \%$ ether/hexanes); IR (neat, $\mathrm{KBr}, \mathrm{cm}^{-1}$ ) 2978, 2939, 2880, 1738, 1732, 1639, 1456, 1377, 1327, 1250, 1188, 1165, 1090, 1059, 993, 922; ${ }^{1} \mathrm{H}$ NMR (500 MHz, $\left.\mathrm{CDCl}_{3}\right) \delta$ (mixture) $5.92-5.80(\mathrm{~m}, 2 \mathrm{H})$, $5.27(\mathrm{dd}, J=17.2,1.4 \mathrm{~Hz}, 1 \mathrm{H}), 5.17(\mathrm{dd}, J=10.5,1.1 \mathrm{~Hz}, 1 \mathrm{H}), 5.06(\mathrm{dd}, J=17.2,1.1 \mathrm{~Hz}, 1 \mathrm{H})$, $5.03(\mathrm{~d}, J=10.3 \mathrm{~Hz}, 1 \mathrm{H}), 4.95-4.88(\mathrm{~m}, 1 \mathrm{H}), 4.60-4.50(\mathrm{~m}, 2 \mathrm{H}), 3.99-3.91(\mathrm{~m}, 1 \mathrm{H}), 3.86-$ $3.77(\mathrm{~m}, 1 \mathrm{H}), 3.09-2.98(\mathrm{~m}, 1 \mathrm{H}), 2.48(\mathrm{p}, J=7.1 \mathrm{~Hz}, 1 \mathrm{H}), 1.98-1.85(\mathrm{~m}, 2 \mathrm{H}), 1.80-1.62(\mathrm{~m}$, 2H), $1.61-1.53(\mathrm{~m}, 1 \mathrm{H}), 1.50-1.39(\mathrm{~m}, 1 \mathrm{H}), 1.19(\mathrm{dd}, J=7.1,0.6 \mathrm{~Hz}, 3 \mathrm{H}), 1.17(\mathrm{dd}, J=6.2,4.6$ $\mathrm{Hz}, 3 \mathrm{H}), 1.06(\mathrm{dd}, J=7.1,0.8 \mathrm{~Hz}, 3 \mathrm{H}) ;{ }^{13} \mathrm{C} \mathrm{NMR}\left(125 \mathrm{MHz}, \mathrm{CDCl}_{3}\right) \delta$ (mixture) 174.4, 173.8, 173.7, 137.4, 137.3, 132.3, 118.0, 115.7, 115.6, 80.5, 80.4, 76.4, 76.3, 69.5, 69.4, 65.0, 45.5, 43.9, 43.8, 42.5, 31.4, 28.5, 20.6, 20.5, 16.7, 16.6, 13.4; ESI-HRMS m/z calcd for $\mathrm{C}_{18} \mathrm{H}_{29} \mathrm{O}_{5}[\mathrm{M}+\mathrm{H}]^{+}$ 325.2010, found 325.2016; ESI-HRMS $\mathrm{m} / \mathrm{z}$ calcd for $\mathrm{C}_{18} \mathrm{H}_{28} \mathrm{NaO}_{5}[\mathrm{M}+\mathrm{Na}]^{+} 347.1829$, found 347.1837 .

But-3-enoic acid 1-methyl-2-\{5-[1-(1-methyl-allyloxycarbonyl)-ethyl]-tetrahydrofuran-2-yl\}ethyl ester (8c). Using the general procedure as described above, alcohol $7 \mathbf{b}(0.009 \mathrm{~g}, 0.04 \mathrm{mmol})$ was reacted to give $0.013 \mathrm{~g}(86 \%)$ of the diester 8c as a colorless oil (mixture of diastereomers) after flash chromatography $\left(\mathrm{SiO}_{2}, 2 \mathrm{~g}, 5 \%\right.$ ether/pentane): TLC $\mathrm{R}_{\mathrm{f}}=0.34$ (20\% ether/pentane); IR (neat, $\mathrm{KBr}, \mathrm{cm}^{-1}$ ) 2978, 2935, 2878, 2359, 2343, 1738, 1732, 1647, 1458, 1377, 1256, 1178, 1090, 1055, 993, 922; ${ }^{1} \mathrm{H} \mathrm{NMR}\left(500 \mathrm{MHz}, \mathrm{CDCl}_{3}\right) \delta$ (mixture) $5.97-5.79(\mathrm{~m}, 2 \mathrm{H}), 5.37(\mathrm{p}, J=5.6 \mathrm{~Hz}$, $1 \mathrm{H}), 5.25(\mathrm{ddd}, J=17.2,3.0,1.0 \mathrm{~Hz}, 1 \mathrm{H}), 5.15(\mathrm{dd}, J=17.5,5.8 \mathrm{~Hz}, 2 \mathrm{H}), 5.03-4.94(\mathrm{~m}, 1 \mathrm{H})$, $4.01(\mathrm{qd}, J=7.1,2.4 \mathrm{~Hz}, 1 \mathrm{H}), 3.92-3.82(\mathrm{~m}, 1 \mathrm{H}), 3.05(\mathrm{~d}, J=6.9 \mathrm{~Hz}, 2 \mathrm{H}), 2.50(\mathrm{pd}, J=7.2,3.0$ $\mathrm{Hz}, 1 \mathrm{H}), 2.04-1.84(\mathrm{~m}, 3 \mathrm{H}), 1.78(\mathrm{ddd}, J=13.2,7.9,5.0 \mathrm{~Hz}, 1 \mathrm{H}), 1.71(\mathrm{ddd}, J=14.0,8.0,5.0 \mathrm{~Hz}$, $1 \mathrm{H}), 1.66-1.58(\mathrm{~m}, 1 \mathrm{H}), 1.55-1.46(\mathrm{~m}, 1 \mathrm{H}), 1.31(\mathrm{dd}, J=6.3,4.1 \mathrm{~Hz}, 3 \mathrm{H}), 1.24(\mathrm{~d}, J=6.3 \mathrm{~Hz}$, $3 \mathrm{H}), 1.10(\mathrm{dd}, J=7.0,2.1 \mathrm{~Hz}, 3 \mathrm{H}) ;{ }^{13} \mathrm{C} \mathrm{NMR}\left(125 \mathrm{MHz}, \mathrm{CDCl}_{3}\right) \delta$ (mixture) 174.2, 174.1, 171.1, 137.9, 137.8, 130.6, 118.5, 115.8, 115.7, 80.6, 80.5, 76.5, 76.4, 70.9, 69.9, 69.8, 45.7, 42.6, 42.5, 39.6, 31.5, 28.6, 28.5, 20.8, 20.0, 19.9, 13.4, 13.3; ESI-HRMS m/z calcd for $\mathrm{C}_{18} \mathrm{H}_{29} \mathrm{O}_{5}[\mathrm{M}+\mathrm{H}]^{+}$ 325.2010, found 325.2005; ESI-HRMS $\mathrm{m} / \mathrm{z}$ calcd for $\mathrm{C}_{18} \mathrm{H}_{28} \mathrm{NaO}_{5}[\mathrm{M}+\mathrm{Na}]^{+} 347.1829$, found 347.1824 . 
2-Methyl-but-3-enoic acid 1-Methyl-2-[5-[1-(1-methyl-allyloxycarbonyl)ethyl]tetrahydrofuran-2yl]ethyl ester (8d). Using the general procedure as described above, alcohol 7b (0.006 g, $0.03 \mathrm{mmol})$ was reacted to give $0.006 \mathrm{~g}(79 \%)$ of the diester $\mathbf{8 d}$ as a colorless oil (mixture of diastereomers) after flash chromatography $\left(\mathrm{SiO}_{2}, 2 \mathrm{~g}, 5 \%\right.$ ether/pentane $\rightarrow 7 \%$ ether/pentane $)$ : TLC $\mathrm{R}_{\mathrm{f}}=0.26(20 \%$ ether/hexanes); IR (neat, $\mathrm{KBr}, \mathrm{cm}^{-1}$ ) 2978, 2935, 2878, 1734, 1458, 1375, 1254, 1188, 1088, 1055 , 993, 920; ${ }^{1} \mathrm{H}$ NMR (500 MHz, $\left.\mathrm{CDCl}_{3}\right) \delta$ (mixture) $5.94-5.79(\mathrm{~m}, 2 \mathrm{H}), 5.41-5.33(\mathrm{~m}, 1 \mathrm{H}), 5.25$ $(\mathrm{ddd}, J=17.3,2.2,1.0 \mathrm{~Hz}, 1 \mathrm{H}), 5.15-5.11(\mathrm{~m}, 1 \mathrm{H}), 5.11-5.06(\mathrm{~m}, 1 \mathrm{H}), 5.00-4.92(\mathrm{~m}, 1 \mathrm{H}), 4.05$ $-3.95(\mathrm{~m}, 1 \mathrm{H}), 3.92-3.80(\mathrm{~m}, 1 \mathrm{H}), 3.09$ (p, $J=7.1 \mathrm{~Hz}, 1 \mathrm{H}), 2.50(\mathrm{pd}, J=7.1,2.8 \mathrm{~Hz}, 1 \mathrm{H}), 2.01-$ $1.88(\mathrm{~m}, 2 \mathrm{H}), 1.82-1.67(\mathrm{~m}, 3 \mathrm{H}), 1.66-1.56(\mathrm{~m}, 1 \mathrm{H}), 1.54-1.45(\mathrm{~m}, 1 \mathrm{H}), 1.33-1.29(\mathrm{~m}, 3 \mathrm{H})$, $1.27-1.20(\mathrm{~m}, 6 \mathrm{H}), 1.12-1.08(\mathrm{~m}, 3 \mathrm{H}) ;{ }^{1} \mathrm{H}$ NMR $\left(500 \mathrm{MHz}, \mathrm{C}_{6} \mathrm{D}_{6}\right) \delta$ (mixture) $6.02-5.92(\mathrm{~m}$, 1H), 5.78 (dddd, $J=17.2,10.5,8.6,5.6 \mathrm{~Hz}, 1 \mathrm{H}), 5.55-5.46(\mathrm{~m}, 1 \mathrm{H}), 5.25-5.15(\mathrm{~m}, 2 \mathrm{H}), 5.04$ (dddd, $J=6.8,5.5,3.0,2.0 \mathrm{~Hz}, 1 \mathrm{H}), 5.00-4.94(\mathrm{~m}, 1 \mathrm{H}), 4.03-3.95(\mathrm{~m}, 1 \mathrm{H}), 3.83-3.70(\mathrm{~m}, 1 \mathrm{H})$, $3.10-2.98(\mathrm{~m}, 1 \mathrm{H}), 2.58-2.44(\mathrm{~m}, 1 \mathrm{H}), 1.68(\mathrm{ddd}, J=16.6,8.1,4.6 \mathrm{~Hz}, 1 \mathrm{H}), 1.62-1.48(\mathrm{~m}, 3 \mathrm{H})$, 1.39 (dd, $J=7.1,0.9 \mathrm{~Hz}, 1 \mathrm{H}), 1.38-1.26(\mathrm{~m}, 2 \mathrm{H}), 1.26-1.16(\mathrm{~m}, 9 \mathrm{H}), 1.04-0.98(\mathrm{~m}, 3 \mathrm{H}) ;{ }^{13} \mathrm{C}$ NMR $\left(125 \mathrm{MHz}, \mathrm{CDCl}_{3}\right) \delta(\mathrm{maJ}=$ or) $174.2,174.0,137.9,137.5,115.8,115.7,80.5,76.5,70.9$, 69.7, 45.7, 44.0, 42.6, 31.6, 28.5, 20.7, 20.0, 16.8, 13.3; ${ }^{13} \mathrm{C} \mathrm{NMR}\left(125 \mathrm{MHz}, \mathrm{C}_{6} \mathrm{D}_{6}\right) \delta$ (major) 173.4, 173.2, 138.4, 138.1, 115.5, 115.4, 80.8, 76.4, 70.7, 69.5, 45.8, 44.3, 42.9, 31.6, 28.5, 20.8, 20.0, 16.9, 13.3; ESI-HRMS m/z calcd for $\mathrm{C}_{19} \mathrm{H}_{31} \mathrm{O}_{5}[\mathrm{M}+\mathrm{H}]^{+}$339.2166, found 339.2157; ESIHRMS $m / z$ calcd for $\mathrm{C}_{19} \mathrm{H}_{30} \mathrm{NaO}_{5}[\mathrm{M}+\mathrm{Na}]^{+} 361.1985$, found 361.1980 .

2,11-Dimethyl-4,10,16-trioxabicyclo[11.2.1]hexadec-6-ene-3,9-dione (9a). To a solution of 0.244 $\mathrm{g}(0.79 \mathrm{mmol}, 1.0 \mathrm{eq})$ of diester $8 \mathrm{a}$ in $316 \mathrm{~mL}$ of $\mathrm{CH}_{2} \mathrm{Cl}_{2}$ was added $0.033 \mathrm{~g}(0.04 \mathrm{mmol}, 5 \mathrm{~mol} \%)$ of Grubbs' 2nd generation catalyst. The reaction mixture was stirred at room temperature for $72 \mathrm{~h}$. After removal of the solvent under reduced pressure the crude product was purified by flash chromatography $\left(\mathrm{SiO}_{2}, 27 \mathrm{~g}, 6 \%\right.$ ether/pentane $\rightarrow 8 \%$ ether/pentane) giving $0.051 \mathrm{~g}(0.16 \mathrm{mmol})$ of starting material 8a (79\% conversion) and $0.141 \mathrm{~g}(81 \%$ based on recovered starting material) of macrocycle 9a (cis/trans ratio 1:5) as a white solid: TLC $\mathrm{R}_{\mathrm{f}}=0.32$ (30\% ether/pentane); mp 65.9$67.3{ }^{\circ} \mathrm{C}$; IR (neat, $\mathrm{KBr}, \mathrm{cm}^{-1}$ ) 2976, 2939, 2878, 1738, 1732, 1462, 1456, 1381, 1327, 1265, 1188 , 1163, 1090, 972; ${ }^{1} \mathrm{H}$ NMR (500 MHz, $\left.\mathrm{CDCl}_{3}\right) \delta$ (major) 6.04 (dtt, $\left.J=15.4,6.8,1.5 \mathrm{~Hz}, 1 \mathrm{H}\right), 5.65$ $(\mathrm{dt}, J=15.8,4.4 \mathrm{~Hz}, 1 \mathrm{H}), 5.13-5.05(\mathrm{~m}, 1 \mathrm{H}), 4.83(\mathrm{ddd}, J=13.6,3.9,1.5 \mathrm{~Hz}, 1 \mathrm{H}), 4.41(\mathrm{ddd}, J=$ 13.6, 4.8, $1.3 \mathrm{~Hz}, 1 \mathrm{H}), 4.06-3.97(\mathrm{~m}, 1 \mathrm{H}), 3.91$ (dt, $J=10.1,6.8 \mathrm{~Hz}, 1 \mathrm{H}), 3.12-3.04(\mathrm{~m}, 1 \mathrm{H})$, $2.96(\mathrm{ddd}, J=16.1,6.8,1.2 \mathrm{~Hz}, 1 \mathrm{H}), 2.42(\mathrm{dq}, J=10.1,7.0 \mathrm{~Hz}, 1 \mathrm{H}), 2.08-1.86(\mathrm{~m}, 3 \mathrm{H}), 1.65$ (ddd, $J=15.3,3.3,1.1 \mathrm{~Hz}, 1 \mathrm{H}), 1.55-1.40(\mathrm{~m}, 2 \mathrm{H}), 1.24(\mathrm{~d}, J=6.5 \mathrm{~Hz}, 3 \mathrm{H}), 1.12(\mathrm{~d}, J=7.0 \mathrm{~Hz}$, $3 \mathrm{H}) ;{ }^{13} \mathrm{C} \mathrm{NMR}\left(125 \mathrm{MHz}, \mathrm{CDCl}_{3}\right) \delta(\mathrm{maJ}=$ or) $174.8,170.7,126.4,126.3,82.2,74.5,68.7,62.4$, $46.6,39.6,38.5,32.1,29.1,18.3,14.6$; $\delta$ (minor) 175.7, 170.6, 130.6, 126.0, 82.3, 75.1, 68.2, 58.1, 46.1, 38.7, 33.9, 31.4, 29.2, 19.3, 14.4; ESI-HRMS m/z calcd for $\mathrm{C}_{15} \mathrm{H}_{23} \mathrm{O}_{5}[\mathrm{M}+\mathrm{H}]^{+} 283.1540$, found 283.1531; ESI-HRMS m/z calcd for $\mathrm{C}_{15} \mathrm{H}_{22} \mathrm{NaO}_{5}[\mathrm{M}+\mathrm{Na}]^{+} 305.1359$, found 305.1352.

2,8,11-Trimethyl-4,10,16-trioxabicyclo[11.2.1]hexadec-6-ene-3,9-dione (9b). To a solution of $0.130 \mathrm{~g}(0.40 \mathrm{mmol}, 1.0 \mathrm{eq})$ of diester $\mathbf{8 b}$ in $161 \mathrm{~mL}$ of $\mathrm{CH}_{2} \mathrm{Cl}_{2}$ was added $0.025 \mathrm{~g}(0.04 \mathrm{mmol}, 10$ mol\%) of Grubbs'-Hoveyda 2nd generation catalyst. The reaction mixture was stirred at room temperature for $48 \mathrm{~h}$. The catalyst was quenched by addition of $1 \mathrm{~mL}$ of ethyl vinyl ether. After removal of the solvent under reduced pressure the crude product was purified by flash 
chromatography $\left(\mathrm{SiO}_{2}, 12 \mathrm{~g}, 5 \%\right.$ ether/pentane $\rightarrow 7 \%$ ether/pentane) giving $0.003 \mathrm{~g}(0.01 \mathrm{mmol})$ of starting material $\mathbf{8 b}$ ( $97 \%$ conversion) and $0.095 \mathrm{~g}(82 \%$ based on recovered starting material) of macrocycle $\mathbf{9 b}$ (mixture of diastereomers, cis/trans ratio 1:5) as a colorless oil: TLC $\mathrm{R}_{\mathrm{f}}=0.14(20 \%$ ether/hexanes); IR (neat, $\mathrm{KBr}, \mathrm{cm}^{-1}$ ) 2976, 2939, 2878, 1738, 1732, 1458, 1379, 1329, 1258, 1188 , 1165, 1092, 1043, 970; ${ }^{1} \mathrm{H}$ NMR $\left(500 \mathrm{MHz} \mathrm{CDCl}_{3}\right) \delta(\mathrm{maJ}=$ or) $6.10(\mathrm{ddt}, J=15.8,6.7,1.6 \mathrm{~Hz}$, $1 \mathrm{H}), 5.61(\mathrm{dtd}, J=15.8,4.4,1.2 \mathrm{~Hz}, 1 \mathrm{H}), 5.08-5.00(\mathrm{~m}, 1 \mathrm{H}), 4.84(\mathrm{ddt}, J=13.5,4.0,1.3 \mathrm{~Hz}, 1 \mathrm{H})$, 4.44 (ddt, $J=13.6,4.4,1.2 \mathrm{~Hz}, 1 \mathrm{H}), 4.09-3.93(\mathrm{~m}, 1 \mathrm{H}), 3.90$ (ddd, $J=17.2,8.2,5.4 \mathrm{~Hz}, 1 \mathrm{H})$, $3.16-3.02(\mathrm{~m}, 1 \mathrm{H}), 2.48-2.38(\mathrm{~m}, 1 \mathrm{H}), 2.05-1.88(\mathrm{~m}, 4 \mathrm{H}), 1.53-1.40(\mathrm{~m}, 2 \mathrm{H}), 1.27-1.22(\mathrm{~m}$, $6 \mathrm{H}), 1.13(\mathrm{~d}, J=7.0 \mathrm{~Hz}, 3 \mathrm{H}) ;{ }^{1} \mathrm{H}$ NMR $\left(500 \mathrm{MHz}, \mathrm{C}_{6} \mathrm{D}_{6}\right) \delta$ (major) $6.32-6.22(\mathrm{~m}, 1 \mathrm{H}), 5.32$ (dtd, $J=15.8,4.2,1.3 \mathrm{~Hz}, 1 \mathrm{H}), 5.12-5.02(\mathrm{~m}, 1 \mathrm{H}), 4.77$ (dddd, $J=13.8,4.0,1.7,1.2 \mathrm{~Hz}, 1 \mathrm{H}), 4.31-$ $4.22(\mathrm{~m}, 1 \mathrm{H}), 3.92-3.77(\mathrm{~m}, 1 \mathrm{H}), 3.77-3.60(\mathrm{~m}, 1 \mathrm{H}), 3.10-3.00(\mathrm{~m}, 1 \mathrm{H}), 2.48-2.38(\mathrm{~m}, 1 \mathrm{H})$, $1.78-1.70(\mathrm{~m}, 1 \mathrm{H}), 1.54-1.42(\mathrm{~m}, 1 \mathrm{H}), 1.41-1.30(\mathrm{~m}, 2 \mathrm{H}), 1.20(\mathrm{~d}, J=7.0 \mathrm{~Hz}, 3 \mathrm{H}), 1.09(\mathrm{~d}, J=$ $6.5 \mathrm{~Hz}, 3 \mathrm{H}), 1.07-0.99(\mathrm{~m}, 2 \mathrm{H}), 0.98(\mathrm{~d}, J=7.0 \mathrm{~Hz}, 3 \mathrm{H}) ;{ }^{13} \mathrm{C} \mathrm{NMR}\left(125 \mathrm{MHz}, \mathrm{CDCl}_{3}\right) \delta$ (major) $174.7,173.8,132.6,124.3,82.2,74.6,68.7,62.3,46.7,43.4,39.6,31.9,29.1,18.6,17.1,14.6 ;{ }^{13} \mathrm{C}$ NMR (125 MHz, $\left.\mathrm{C}_{6} \mathrm{D}_{6}\right) \delta$ (major) 173.8, 173.0, 132.4, 124.6, 82.2, 74.5, 68.5, 62.0, 46.8, 43.7, 39.7, 32.0, 29.1, 18.4, 16.8, 14.5; ESI-HRMS m/z calcd for $\mathrm{C}_{16} \mathrm{H}_{25} \mathrm{O}_{5}[\mathrm{M}+\mathrm{H}]^{+} 297.1697$, found 297.1701; ESI-HRMS $m / z$ calcd for $\mathrm{C}_{16} \mathrm{H}_{24} \mathrm{NaO}_{5}[\mathrm{M}+\mathrm{Na}]^{+} 319.1516$, found 319.1525 .

2,5,11-Trimethyl-4,10,16-trioxabicyclo[11.2.1]hexadec-6-ene-3,9-dione (9c). To a solution of $0.009 \mathrm{~g}(0.03 \mathrm{mmol}, 1.0 \mathrm{eq})$ of diester $8 \mathrm{c}$ in $11 \mathrm{~mL}$ of $\mathrm{CH}_{2} \mathrm{Cl}_{2}$ was added $0.003 \mathrm{~g}(0.006 \mathrm{mmol}, 20$ mol\%) of Grubbs'-Hoveyda 2nd generation catalyst. The reaction mixture was stirred at room temperature for $48 \mathrm{~h}$. The catalyst was quenched by addition of $0.5 \mathrm{~mL}$ of ethyl vinyl ether. After removal of the solvent under reduced pressure the crude product was purified by flash chromatography ( $\mathrm{SiO}_{2}, 1 \mathrm{~g}, 5 \%$ ether/pentane $\rightarrow 7 \%$ ether/pentane) giving $0.0037 \mathrm{~g}(0.01 \mathrm{mmol})$ of starting material 8c (97\% conversion) and $0.095 \mathrm{~g}$ (82\% based on recovered starting material) of macrocycle 9c (mixture of diastereomers, cis/trans ratio 1:5) as a colorless oil: IR (neat, $\mathrm{KBr}, \mathrm{cm}^{-1}$ ) 2972, 2926, 2854, 1738, 1732, 1717, 1456, 1377, 1265, 1190, 1092, 972; ${ }^{1} \mathrm{H}$ NMR (500 MHz, $\left.\mathrm{CDCl}_{3}\right) \delta$ (major) 6.03 (ddd, $\left.J=21.7,14.8,6.8 \mathrm{~Hz}, 1 \mathrm{H}\right), 5.58-5.49(\mathrm{~m}, 1 \mathrm{H}), 5.17-4.98(\mathrm{~m}, 1 \mathrm{H})$, $4.12-3.96(\mathrm{~m}, 2 \mathrm{H}), 3.96-3.84(\mathrm{~m}, 1 \mathrm{H}), 3.23-2.86(\mathrm{~m}, 3 \mathrm{H}), 2.38-2.25(\mathrm{~m}, 1 \mathrm{H}), 2.11-1.87(\mathrm{~m}$, $2 \mathrm{H}), 1.74-1.54(\mathrm{~m}, 2 \mathrm{H}), 1.54-1.38(\mathrm{~m}, 1 \mathrm{H}), 1.36-1.26(\mathrm{~m}, 6 \mathrm{H}), 1.13(\mathrm{~d}, J=6.9 \mathrm{~Hz}, 3 \mathrm{H}) ;{ }^{13} \mathrm{C}$ NMR (125 MHz, $\left.\mathrm{CDCl}_{3}\right) \delta$ (major) 175.4, 170.9, 132.7, 124.9, 82.6, 74.5, 68.8, 64.8, 46.1, 38.1, 32.1, 29.9, 29.0, 20.4, 17.8, 14.7; ESI-HRMS m/z calcd for $\mathrm{C}_{16} \mathrm{H}_{25} \mathrm{O}_{5}[\mathrm{M}+\mathrm{H}]^{+} 297.1697$, found 297.1683; ESI-HRMS $m / z$ calcd for $\mathrm{C}_{16} \mathrm{H}_{24} \mathrm{NaO}_{5}[\mathrm{M}+\mathrm{Na}]^{+} 319.1516$, found 319.1499 .

2,5,8,11-Tetramethyl-4,10,16-trioxabicyclo[11.2.1]hexadec-6-ene-3,9-dione (9d). To a solution of $0.013 \mathrm{~g}(0.03 \mathrm{mmol}, 1.0 \mathrm{eq})$ of diester $8 \mathbf{d}$ in $12 \mathrm{~mL}$ of $\mathrm{CH}_{2} \mathrm{Cl}_{2}$ was added $0.004 \mathrm{~g}(0.006 \mathrm{mmol}$, $20 \mathrm{~mol} \%$ ) of Grubbs'-Hoveyda 2 nd generation catalyst. The reaction mixture was stirred at room temperature for $5 \mathrm{~d}$. The catalyst was quenched by addition of $0.5 \mathrm{~mL}$ of ethyl vinyl ether. After removal of the solvent under reduced pressure the crude product was purified by flash chromatography ( $\mathrm{SiO}_{2}, 1 \mathrm{~g}, 5 \%$ ether/pentane $\rightarrow 7 \%$ ether/pentane) giving $0.0014 \mathrm{~g}(0.01 \mathrm{mmol})$ of starting material 8 (84\% conversion) and $0.0046 \mathrm{~g}$ (52\% based on recovered starting material) of macrocycle 9d (mixture of diastereomers, cis/trans ratio 1:5) as a colorless oil: ${ }^{1} \mathrm{H}$ NMR (500 MHz, $\left.\mathrm{CDCl}_{3}\right) \delta$ (major) $6.14(\mathrm{ddd}, J=15.7,6.0,1.3 \mathrm{~Hz}, 1 \mathrm{H}), 5.73-5.63(\mathrm{~m}, 1 \mathrm{H}), 5.17$ (ddq, $J=10.1$, 
6.6, $3.5 \mathrm{~Hz}, 1 \mathrm{H}), 4.14-3.83(\mathrm{~m}, 3 \mathrm{H}), 3.34(\mathrm{dq}, J=11.0,7.0 \mathrm{~Hz}, 1 \mathrm{H}), 3.13-3.08(\mathrm{~m}, 1 \mathrm{H}), 2.46-$ $2.37(\mathrm{~m}, 1 \mathrm{H}), 2.09-1.86(\mathrm{~m}, 3 \mathrm{H}), 1.69-1.60(\mathrm{~m}, 1 \mathrm{H}), 1.52-1.40(\mathrm{~m}, 1 \mathrm{H}), 1.31(\mathrm{~d}, J=6.5 \mathrm{~Hz}$, $3 \mathrm{H}), 1.28-1.22(\mathrm{~m}, 6 \mathrm{H}), 1.12(\mathrm{~d}, J=6.5 \mathrm{~Hz}, 3 \mathrm{H})$; ESI-HRMS $\mathrm{m} / \mathrm{z}$ calcd for $\mathrm{C}_{17} \mathrm{H}_{27} \mathrm{O}_{5}[\mathrm{M}+\mathrm{H}]^{+}$ 311.1853, found 311.1834; ESI-HRMS $\mathrm{m} / \mathrm{z}$ calcd for $\mathrm{C}_{17} \mathrm{H}_{26} \mathrm{NaO}_{5}[\mathrm{M}+\mathrm{Na}]^{+} 333.1672$, found 333.1657.

\section{General TBDPS protection procedure}

To a $0.2 \mathrm{M}$ solution of monoprotected diol 11 or 12 (1.0 eq) in $\mathrm{CH}_{2} \mathrm{Cl}_{2}$ were added triethylamine (4.0 eq), TBDPSCl (1.3 eq) and a catalytic amount of DMAP. Resulting mixture was stirred at room temperature for $4 \mathrm{hr}$. After evaporation of the solvent under reduced pressure the crude product was purified by flash chromatography $\left(\mathrm{SiO}_{2}, 1: 25\right.$ ratio of the crude product to silica gel, $1 \%$ ether/pentane $\rightarrow 2 \%$ ether/pentane).

(2S)-tert-Butyl-[2-(4-methoxybenzyloxy)but-3-enyloxy]diphenylsilane. Using the general procedure as described above, (2S)-2-(4-methoxy-benzyloxy)-but-3-en-1-ol (3.68g, $17.8 \mathrm{mmol})$ was reacted to give a colorless oil $(1.77 \mathrm{~g}, 81 \%$ over 2 steps $)$ after chromatography: TLC $\mathrm{R}_{\mathrm{f}}=0.20(10 \%$ ether/hexanes); IR (neat, $\mathrm{KBr}, \mathrm{cm}^{-1}$ ) 2955, 2930, 2856, 1612, 1514, 1464, 1427, 1248, 1113, 1088, 1038; ${ }^{1} \mathrm{H}$ NMR $\left(500 \mathrm{MHz}, \mathrm{CDCl}_{3}\right) \delta 7.86-7.77(\mathrm{~m}, 4 \mathrm{H}), 7.54-7.48(\mathrm{~m}, 2 \mathrm{H}), 7.45(\mathrm{t}, J=7.2 \mathrm{~Hz}$, 4H), $7.38(\mathrm{~d}, J=8.6 \mathrm{~Hz}, 2 \mathrm{H}), 6.96(\mathrm{~d}, J=8.6 \mathrm{~Hz}, 2 \mathrm{H}), 5.88(\mathrm{ddd}, J=17.4,10.4,7.0 \mathrm{~Hz}, 1 \mathrm{H}), 5.39$ $(\mathrm{dd}, J=17.3,1.2 \mathrm{~Hz}, 1 \mathrm{H}), 5.35(\mathrm{~d}, J=10.4 \mathrm{~Hz}, 1 \mathrm{H}), 4.69(\mathrm{~d}, J=11.6 \mathrm{~Hz}, 1 \mathrm{H}), 4.51(\mathrm{~d}, J=11.6$ $\mathrm{Hz}, 1 \mathrm{H}), 4.05(\mathrm{dd}, J=12.0,6.5 \mathrm{~Hz}, 1 \mathrm{H}), 3.91(\mathrm{dd}, J=10.5,6.6 \mathrm{~Hz}, 1 \mathrm{H}), 3.87(\mathrm{~s}, 3 \mathrm{H}), 3.78(\mathrm{dd}, J=$ 10.5, $5.0 \mathrm{~Hz}, 1 \mathrm{H}), 1.19(\mathrm{~s}, 9 \mathrm{H}) ;{ }^{13} \mathrm{C}$ NMR $\left(125 \mathrm{MHz}, \mathrm{CDCl}_{3}\right) \delta 159.1,136.3,135.8,133.8,133.7$, $130.9,129.7,129.6,129.3,127.7,118.1,113.8,80.9,70.4,66.9,55.3,27.0,19.4 ;[\alpha]_{\mathrm{D}}+10.8,(\mathrm{c}$ 1.00, $\mathrm{CHCl}_{3}$ ); ESI-HRMS m/z calcd for $\mathrm{C}_{28} \mathrm{H}_{34} \mathrm{NaO}_{3} \mathrm{Si}[\mathrm{M}+\mathrm{Na}]^{+} 469.2169$, found 469.2170.

(2R)-tert-Butyl-[2-(4-methoxybenzyloxy)but-3-enyloxy]diphenylsilane. Using the general procedure as described above, (2R)-2-(4-methoxy-benzyloxy)-but-3-en-1-ol (0.5389 g, $2.59 \mathrm{mmol})$ was reacted to give a colorless oil $(0.698 \mathrm{~g}, 79 \%$ over 2 steps $)$ after chromatography: TLC $\mathrm{R}_{\mathrm{f}}=0.20$ (10\% ether/hexanes); IR (neat, $\left.\mathrm{KBr}, \mathrm{cm}^{-1}\right)$ 2957, 2932, 2858, 1612, 1514, 1464, 1427, 1248, 1113 , 1088, 1038; ${ }^{1} \mathrm{H}$ NMR (500 MHz, $\left.\mathrm{CDCl}_{3}\right) \delta 7.72$ (ddd, $\left.J=7.0,5.2,2.3 \mathrm{~Hz}, 4 \mathrm{H}\right), 7.45$ (t, $J=7.3 \mathrm{~Hz}$, 2H), 7.39 (t, $J=7.2 \mathrm{~Hz}, 4 \mathrm{H}), 7.31(\mathrm{~d}, J=8.5 \mathrm{~Hz}, 2 \mathrm{H}), 6.90(\mathrm{~d}, J=8.6 \mathrm{~Hz}, 2 \mathrm{H}), 5.81$ (dddd, $J=$ 10.2, 9.3, 7.1, 2.2 Hz, 1H), $5.32(\mathrm{~d}, J=18.7 \mathrm{~Hz}, 1 \mathrm{H}), 5.29(\mathrm{~d}, J=9.2 \mathrm{~Hz}, 1 \mathrm{H}), 4.62(\mathrm{~d}, J=11.6 \mathrm{~Hz}$, $1 \mathrm{H}), 4.45(\mathrm{~d}, J=11.6 \mathrm{~Hz}, 1 \mathrm{H}), 3.98(\mathrm{dd}, J=12.0,6.4 \mathrm{~Hz}, 1 \mathrm{H}), 3.86-3.80(\mathrm{~m}, 1 \mathrm{H}), 3.83(\mathrm{~s}, 3 \mathrm{H})$, 3.69 (ddd, $J=10.5,5.0,2.5 \mathrm{~Hz}, 1 \mathrm{H}), 1.11(\mathrm{bs}, J=2.4 \mathrm{~Hz}, 9 \mathrm{H}) ;{ }^{13} \mathrm{C} \mathrm{NMR}\left(125 \mathrm{MHz}, \mathrm{CDCl}_{3}\right) \delta$ 159.2, 136.3, 135.8, 133.8, 133.7, 131.0, 129.7, 129.7, 129.4, 127.7, 118.2, 113.8, 80.9, 70.5, 67.0, 55.4, 27.0, 19.4; $[\alpha]_{\mathrm{D}}-9.4$, (c 1.00, $\mathrm{CHCl}_{3}$ ); ESI-HRMS m/z calcd for $\mathrm{C}_{28} \mathrm{H}_{34} \mathrm{NaO}_{3} \mathrm{Si}[\mathrm{M}+\mathrm{Na}]^{+}$ 469.2169, found 469.2151.

\section{General PMB deprotection procedure}

Ethers (1.0 eq) was dissolved in ice-cold $10 \%$ (by volume) solution of trifluoroacetic acid in $\mathrm{CH}_{2} \mathrm{Cl}_{2}$ (16.5 eq). The cooling bath was removed and the resulting solution was stirred at room temperature for $15 \mathrm{~min}$. Then saturated aqueous $\mathrm{NaHCO}_{3}$ (2.8 times the volume of the reaction mixture) was added, the organic layer was separated and the aqueous layer was extracted with $\mathrm{CH}_{2} \mathrm{Cl}_{2}$. The 
combined organic extracts were dried over $\mathrm{MgSO}_{4}$ and concentrated under reduced pressure. The crude product was purified by flash chromatography $\left(\mathrm{SiO}_{2}, 1: 100\right.$ ratio of the crude product to silica gel, $2 \%$ ether/pentane $\rightarrow 3 \%$ ether/pentane).

(2S)-1-(tert-Butyldiphenylsilanyloxy)but-3-en-2-ol (13). Using the general procedure as described above, (2S)-tert-butyl-[2-(4-methoxy-benzyloxy)-but-3-enyloxy]-diphenyl-silane (0.403 g, 0.90 mmol) was reacted to give 13 as a colorless oil $(0.214 \mathrm{~g}, 73 \%)$ after chromatography: TLC $\mathrm{R}_{\mathrm{f}}=$ 0.45 (20\% ether/pentane); IR (neat, $\mathrm{KBr}, \mathrm{cm}^{-1}$ ) 3443 (broad), 2959, 2930, 2858, 1472, 1427, 1113, 1070, 989, 926; ${ }^{1} \mathrm{H}$ NMR $\left(500 \mathrm{MHz}, \mathrm{CDCl}_{3}\right) \delta 7.69(\mathrm{dd}, J=7.9,1.4 \mathrm{~Hz}, 4 \mathrm{H}), 7.44(\mathrm{ddt}, J=21.6$, $14.2,4.0 \mathrm{~Hz}, 6 \mathrm{H}), 5.81(\mathrm{ddd}, J=17.2,10.6,5.6 \mathrm{~Hz}, 1 \mathrm{H}), 5.34(\mathrm{dt}, J=17.3,1.5 \mathrm{~Hz}, 1 \mathrm{H}), 5.18$ (dt, $J$ $=10.6,1.4 \mathrm{~Hz}, 1 \mathrm{H}), 4.30-4.23(\mathrm{~m}, 1 \mathrm{H}), 3.72(\mathrm{dd}, J=10.2,3.8 \mathrm{~Hz}, 1 \mathrm{H}), 3.58(\mathrm{dd}, J=10.2,7.5$ $\mathrm{Hz}, 1 \mathrm{H}), 2.61$ (bs, $1 \mathrm{H}), 1.10(\mathrm{~s}, 9 \mathrm{H}) ;{ }^{13} \mathrm{C} \mathrm{NMR}\left(125 \mathrm{MHz}, \mathrm{CDCl}_{3}\right) \delta 136.8,135.7,135.6,133.2$, 133.1, 130.0, 127.9, 116.6, 73.1, 67.8, 27.0, 19.4; $[\alpha]_{\mathrm{D}}-4.7$, (c 1.00, $\left.\mathrm{CHCl}_{3}\right)$; ESI-HRMS m/z calcd for $\mathrm{C}_{20} \mathrm{H}_{26} \mathrm{NaO}_{2} \mathrm{Si}[\mathrm{M}+\mathrm{Na}]^{+} 349.1594$, found 349.1581.

(2R)-1-(tert-Butyldiphenylsilanyloxy)but-3-en-2-ol (14). Using the general procedure as described above, (2R)-tert-Butyl-[2-(4-methoxybenzyloxy)but-3-enyloxy]diphenylsilane 52b (0.481 g, 1.08 mmol) was reacted to give $45 \mathrm{~b}$ as a colorless oil $(0.352 \mathrm{~g}, 85 \%)$ after chromatography: TLC $\mathrm{R}_{\mathrm{f}}=$ 0.45 (20\% ether/pentane); IR (neat, $\mathrm{KBr}, \mathrm{cm}^{-1}$ ) 3439 (broad), 2959, 2930, 2858, 1472, 1427, 1113, 1068, 989, 926; ${ }^{1} \mathrm{H}$ NMR (500 MHz, $\left.\mathrm{CDCl}_{3}\right) \delta 7.67(\mathrm{dd}, J=7.9,1.4 \mathrm{~Hz}, 4 \mathrm{H}), 7.48-7.37(\mathrm{~m}, 6 \mathrm{H})$, $5.80(\mathrm{ddd}, J=17.2,10.6,5.6 \mathrm{~Hz}, 1 \mathrm{H}), 5.32(\mathrm{dt}, J=17.3,1.5 \mathrm{~Hz}, 1 \mathrm{H}), 5.17(\mathrm{dt}, J=10.6,1.4 \mathrm{~Hz}$, $1 \mathrm{H}), 4.30-4.21(\mathrm{~m}, 1 \mathrm{H}), 3.71(\mathrm{dd}, J=10.2,3.8 \mathrm{~Hz}, 1 \mathrm{H}), 3.56(\mathrm{dd}, J=10.2,7.5 \mathrm{~Hz}, 1 \mathrm{H}), 2.61(\mathrm{~d}, J$ $=3.8 \mathrm{~Hz}, 1 \mathrm{H}), 1.11-1.06(\mathrm{~m}, 9 \mathrm{H}) ;{ }^{13} \mathrm{C} \mathrm{NMR}\left(125 \mathrm{MHz}, \mathrm{CDCl}_{3}\right) \delta 136.7,135.7,135.6,133.3$, 133.2, 130.0, 127.9, 116.7, 73.2, 67.8, 27.0, 19.4; $[\alpha]_{\mathrm{D}}+4.8,\left(\mathrm{c} 1.00, \mathrm{CHCl}_{3}\right)$; ESI-HRMS m/z calcd for $\mathrm{C}_{20} \mathrm{H}_{26} \mathrm{NaO}_{2} \mathrm{Si}[\mathrm{M}+\mathrm{Na}]^{+} 349.1594$, found 349.1582 .

2-[5-(2-Azidopropyl)tetrahydrofuran-2-yl]propionic acid methyl ester (18). Methyl (-)nonactate (-)-15 (0.821 g, $3.8 \mathrm{mmol}, 1.0 \mathrm{eq})$ and triethylamine $(1.1 \mathrm{~mL}, 7.6 \mathrm{mmol}, 2.0 \mathrm{eq})$ were dissolved in $19 \mathrm{~mL}$ of anhydrous $\mathrm{CH}_{2} \mathrm{Cl}_{2}$ and cooled to $0{ }^{\circ} \mathrm{C}$. Mesyl chloride $(0.38 \mathrm{~mL}, 4.9 \mathrm{mmol}$, $1.3 \mathrm{eq}$ ) was added dropwise, and the resulting solution was stirred at $0{ }^{\circ} \mathrm{C}$ under argon for $2 \mathrm{hr}$. Then, it was poured into ice-cold $\mathrm{HCl}(1.0 \mathrm{M}, 7.6 \mathrm{~mL})$ and stirred vigorously. The aqueous layer was separated and extracted with $\mathrm{CH}_{2} \mathrm{Cl}_{2}(3 * 8 \mathrm{~mL})$. The combined organic extracts were washed with saturated $\mathrm{NaHCO}_{3}$, dried over $\mathrm{MgSO}_{4}$ and concentrated to give crude mesylate. A solution of the crude mesylate in $12.7 \mathrm{~mL}$ of DMF was placed in a sealed tube equipped with a stir bar, $\mathrm{NaN}_{3}$ (0.493 g, $7.6 \mathrm{mmol}, 2.0 \mathrm{eq})$ was added, and the resulting mixture was stirred at $50{ }^{\circ} \mathrm{C}$ under argon for $18 \mathrm{~h}$. The reaction mixture was diluted with $140 \mathrm{~mL}$ of water, and poured into $200 \mathrm{~mL}$ of diethyl ether. The organic layer was separated, and the aqueous layer was extracted with ether $(3 * 150 \mathrm{~mL})$. The combined organic extracts were dried over $\mathrm{MgSO}_{4}$ and concentrated under reduced pressure to give $1.05 \mathrm{~g}$ of the crude azide. Flash chromatography $\left(\mathrm{SiO}_{2}, 100 \mathrm{~g}, 4 \%\right.$ ether/pentane $\rightarrow 10 \%$ ether/pentane) afforded $0.8690 \mathrm{~g}(95 \%)$ of the azidoester 56 as a colorless oil (mixture of diastereomers): TLC $\mathrm{R}_{\mathrm{f}}=0.19$ (10\% ether/pentane); IR (neat, $\mathrm{KBr}, \mathrm{cm}^{-1}$ ) 2974, 2949, 2880, 2359, 2341, 2102, 1748, 1460, 1435, 1379, 1331, 1256, 1200, 1165, 1090, 1061; ${ }^{1} \mathrm{H}$ NMR (500 MHz, $\left.\mathrm{CDCl}_{3}\right) \delta$ (mixture) $3.98(\mathrm{qd}, J=6.9,1.8 \mathrm{~Hz}, 1 \mathrm{H}), 3.94-3.86(\mathrm{~m}, 1 \mathrm{H}), 3.65(\mathrm{~d}, J=2.2 \mathrm{~Hz}, 3 \mathrm{H})$, $3.57-3.47(\mathrm{~m}, 1 \mathrm{H}), 2.54-2.42(\mathrm{~m}, 1 \mathrm{H}), 2.03-1.88(\mathrm{~m}, 2 \mathrm{H}), 1.80(\mathrm{tdd}, J=8.6,7.3,2.1 \mathrm{~Hz}, 1 \mathrm{H})$, 
$1.67-1.55(\mathrm{~m}, 1 \mathrm{H}), 1.55-1.43(\mathrm{~m}, 2 \mathrm{H}), 1.23(\mathrm{dd}, J=6.5,2.0 \mathrm{~Hz}, 3 \mathrm{H}), 1.08(\mathrm{dd}, J=7.0,2.0 \mathrm{~Hz}$, $3 \mathrm{H}) ;{ }^{13} \mathrm{C}$ NMR $\left(125 \mathrm{MHz}, \mathrm{CDCl}_{3}\right) \delta$ (mixture) $175.2,80.7,76.3,55.4,51.6,45.4,42.1,31.1,28.5$, 19.3, 13.5; $[\alpha]_{\mathrm{D}}-0.4,\left(\mathrm{c} 1.00, \mathrm{CHCl}_{3}\right)$; ESI-HRMS m/z calcd for $\mathrm{C}_{11} \mathrm{H}_{19} \mathrm{~N}_{3} \mathrm{NaO}_{3}[\mathrm{M}+\mathrm{Na}]^{+}$ 264.1319, found 264.1319.

2-[5-(2-Azidopropyl)tetrahydrofuran-2-yl]propionic acid (19). To a solution of the azidoester 18 $(0.869 \mathrm{~g}, 3.6 \mathrm{mmol}, 1.0 \mathrm{eq})$ in $18 \mathrm{~mL}$ of THF and $6 \mathrm{~mL}$ of $\mathrm{MeOH}$ was added $36 \mathrm{~mL}$ of $\mathrm{KOH}(1.0$ M). The reaction mixture was stirred at room temperature for $20 \mathrm{~h}$. Then, it was concentrated under reduced pressure, partially dissolved in ether and treated with $18 \mathrm{~mL}$ of ice-cold $\mathrm{HCl}(2.0 \mathrm{M})$. After vigorous stirring the organic layer was separated and the aqueous layer was extracted with ether $(3 * 20 \mathrm{~mL})$. The combined organic extracts were dried over $\mathrm{MgSO}_{4}$ and concentrated under reduced pressure to give $0.792 \mathrm{~g}$ (97\%) of carboxylic acid $\mathbf{1 9}$ as a colorless oil (mixture of diastereomers), which was used without further purification. TLC $\mathrm{R}_{\mathrm{f}}=0.26$ (60\% ether / pentane); IR (neat, $\mathrm{KBr}$, $\left.\mathrm{cm}^{-1}\right) 3100$ (broad), 2974, 2920, 2359, 2341, 2102, 1711, 1462, 1379, 1250, 1090, 667; ${ }^{1} \mathrm{H}$ NMR $\left(500 \mathrm{MHz}, \mathrm{CDCl}_{3}\right) \delta$ (mixture) $10.89(\mathrm{~s}, 1 \mathrm{H}), 4.09-3.95(\mathrm{~m}, 2 \mathrm{H}), 3.57(\mathrm{~h}, J=6.6 \mathrm{~Hz}, 1 \mathrm{H}), 2.58-$ $2.48(\mathrm{~m}, 1 \mathrm{H}), 2.11-1.97(\mathrm{~m}, 2 \mathrm{H}), 1.92-1.80(\mathrm{~m}, 1 \mathrm{H}), 1.73-1.61(\mathrm{~m}, 1 \mathrm{H}), 1.61-1.50(\mathrm{~m}, 2 \mathrm{H})$, $1.29(\mathrm{~d}, J=6.5 \mathrm{~Hz}, 3 \mathrm{H}), 1.17(\mathrm{~d}, J=7.0 \mathrm{~Hz}, 3 \mathrm{H}) ;{ }^{13} \mathrm{C}$ NMR $\left(125 \mathrm{MHz}, \mathrm{CDCl}_{3}\right) \delta$ (mixture) 179.4, 80.5, 76.9, 55.4, 45.3, 42.1, 31.1, 28.9, 19.5, 13.5; $[\alpha]_{\mathrm{D}}+0.1,\left(\mathrm{c} 1.00, \mathrm{CHCl}_{3}\right)$, ESI-HRMS m/z calcd for $\mathrm{C}_{10} \mathrm{H}_{17} \mathrm{~N}_{3} \mathrm{NaO}_{3}[\mathrm{M}+\mathrm{Na}]^{+} 250.1162$, found 250.1164 .

\section{General esterification procedure}

A $0.4 \mathrm{M}$ solution of 8-azido-nonactic acid 19 (1.6 eq) in anhydrous benzene was placed in a twonecked flask equipped with a stir bar and a drying tube. Oxalyl chloride (2.8 eq) was added dropwise via syringe, and the reaction mixture was stirred at room temperature until bubbling completely ceased (approx. $6 \mathrm{~h}$ ). Benzene and the excess of oxalyl chloride were removed under reduced pressure leaving crude acid chloride as a yellow oil, which was dissolved in anhydrous $\mathrm{CH}_{2} \mathrm{Cl}_{2}$ to make a $1.0 \mathrm{M}$ solution of 8-azidononactic acid chloride. A $0.2 \mathrm{M}$ solution of monoprotected diol 13 or 14 (1.0 eq) in $\mathrm{CH}_{2} \mathrm{Cl}_{2}$ was cooled down to $0{ }^{\circ} \mathrm{C}$ and $\mathrm{N}$-methylmorpholine (2.0 eq) was added in one portion followed by a dropwise addition of a freshly prepared $1.0 \mathrm{M}$ solution of the acid chloride (1.6 eq) and a catalytic amount of DMAP. Reaction mixture was stirred at $0{ }^{\circ} \mathrm{C}$ for $1 \mathrm{~h}$ and another $6 \mathrm{~h}$ at room temperature under argon. After evaporation of the solvent under reduced pressure, the residue was re-dissolved in an ice-cold 3:1 emulsion of ether and $1.0 \mathrm{M}$ $\mathrm{HCl}(2.0 \mathrm{eq}$ ). After vigorous stirring the organic layer was separated and the aqueous layer was extracted with ether. The combined organic extracts were dried over $\mathrm{MgSO}_{4}$ and concentrated under reduced pressure. The crude product was purified by flash chromatography $\left(\mathrm{SiO}_{2}, 1: 85\right.$ ratio of the crude product to silica gel, $3 \%$ ether/pentane $\rightarrow 5 \%$ ether/pentane).

2-[5-(2-Azidopropyl)tetrahydrofuran-2-yl]propionic acid 1-(tert-butyldiphenylsilanyloxymethyl)allyl ester (20a). Using the general procedure as described above, (2S)-1-(tert-butyldiphenylsilanyloxy)but-3-en-2-ol $(0.497 \mathrm{~g}, 1.52 \mathrm{mmol})$ was reacted to give $0.722 \mathrm{~g}(88 \%)$ of the azidoester 20a as a colorless oil (mixture of diastareomers) after chromatography: TLC $\mathrm{R}_{\mathrm{f}}=0.19$ (10\% ether/pentane); IR (neat, $\mathrm{KBr}, \mathrm{cm}^{-1}$ ) 2959, 2932, 2858, 2100, 1738, 1427, 1252, 1188, 1165, 1113, 1090, 997, 935; ${ }^{1} \mathrm{H}$ NMR $\left(500 \mathrm{MHz}, \mathrm{CDCl}_{3}\right) \delta$ (mixture) $7.70-7.64(\mathrm{~m}, 4 \mathrm{H}), 7.46-7.36(\mathrm{~m}, 6 \mathrm{H}), 5.86-5.75(\mathrm{~m}$, 
$1 \mathrm{H}), 5.43(\mathrm{ddd}, J=17.1,11.1,5.9 \mathrm{~Hz}, 1 \mathrm{H}), 5.32(\mathrm{dd}, J=17.3,1.0 \mathrm{~Hz}, 1 \mathrm{H}), 5.20(\mathrm{ddd}, J=10.8$, 2.7, $1.3 \mathrm{~Hz}, 1 \mathrm{H}), 4.07$ (ddd, $J=20.0,14.6,7.1 \mathrm{~Hz}, 1 \mathrm{H}), 4.00-3.86(\mathrm{~m}, 1 \mathrm{H}), 3.80-3.73(\mathrm{~m}, 1 \mathrm{H})$, $3.73-3.67(\mathrm{~m}, 1 \mathrm{H}), 3.60-3.48(\mathrm{~m}, 1 \mathrm{H}), 2.59(\mathrm{td}, J=14.6,7.3 \mathrm{~Hz}, 1 \mathrm{H}), 2.07-1.89(\mathrm{~m}, 2 \mathrm{H}), 1.89$ $-1.76(\mathrm{~m}, 1 \mathrm{H}), 1.74-1.59(\mathrm{~m}, 1 \mathrm{H}), 1.59-1.43(\mathrm{~m}, 2 \mathrm{H}), 1.23(\mathrm{dd}, J=12.0,6.5 \mathrm{~Hz}, 3 \mathrm{H}), 1.13(\mathrm{dd}$, $J=10.3,7.1 \mathrm{~Hz}, 3 \mathrm{H}), 1.04(\mathrm{~s}, 9 \mathrm{H}) ;{ }^{13} \mathrm{C} \mathrm{NMR}\left(125 \mathrm{MHz}, \mathrm{CDCl}_{3}\right) \delta$ (mixture) 174.3, 173.8, 135.8, $135.7,133.7,133.6,133.5,133.4,129.9,129.8,127.8,117.9,117.8,80.7,80.3,76.3,76.2$, 75.1, $75.0,65.5,65.4,55.4,45.6,45.5,42.1,42.0,31.3,28.4,28.2,26.9,26.8,19.4,19.2,13.3,13.2 ;[\alpha]_{\mathrm{D}}$ -10.6, (c 1.01, $\mathrm{CHCl}_{3}$ ); ESI-HRMS $\mathrm{m} / \mathrm{z}$ calcd for $\mathrm{C}_{30} \mathrm{H}_{42} \mathrm{~N}_{3} \mathrm{O}_{4} \mathrm{Si}[\mathrm{M}+\mathrm{H}]^{+}$536.2939, found 536.2929; ESI-HRMS m/z calcd for $\mathrm{C}_{30} \mathrm{H}_{41} \mathrm{~N}_{3} \mathrm{NaO}_{4} \mathrm{Si}[\mathrm{M}+\mathrm{Na}]^{+}$558.2759, found 558.2754.

2-[5-(2-Azidopropyl)tetrahydrofuran-2-yl]propionic acid 1-(tert-butyl-diphenylsilanyloxymethyl)allyl ester (20b). Using the general procedure as described above, (2R)-1-(tert-butyldiphenylsilanyloxy)but-3-en-2-ol 45b (0.109 g, $0.34 \mathrm{mmol})$ was reacted to give $0.163 \mathrm{~g}(91 \%)$ of the azidoester $58 \mathrm{~b}$ as a colorless oil (mixture of diastereomers) after chromatography: TLC $\mathrm{R}_{\mathrm{f}}=0.19$ (10\% ether/pentane); IR (neat, KBr, cm ${ }^{-1}$ ) 2959, 2932, 2858, 2100, 1736, 1427, 1250, 1188, 1163, 1113, 1090, 997, 935; ${ }^{1} \mathrm{H}$ NMR $\left(500 \mathrm{MHz}, \mathrm{CDCl}_{3}\right) \delta$ (mixture) $7.70-7.64(\mathrm{~m}, 4 \mathrm{H}), 7.46-7.35(\mathrm{~m}, 6 \mathrm{H}), 5.81(\mathrm{dq}, J=$ $10.7,5.8 \mathrm{~Hz}, 1 \mathrm{H}), 5.48-5.38(\mathrm{~m}, 1 \mathrm{H}), 5.32(\mathrm{~d}, J=17.3 \mathrm{~Hz}, 1 \mathrm{H}), 5.20(\mathrm{dd}, J=10.7,1.2 \mathrm{~Hz}, 1 \mathrm{H})$, $4.13-4.00$ (m, 1H), $3.95-3.84(\mathrm{~m}, 1 \mathrm{H}), 3.81-3.73(\mathrm{~m}, 1 \mathrm{H}), 3.70$ (ddd, $J=10.7,9.1,4.5 \mathrm{~Hz}, 1 \mathrm{H})$, 3.55 (qd, $J=13.4,6.8 \mathrm{~Hz}, 1 \mathrm{H}), 2.59(\mathrm{dp}, J=14.2,7.2 \mathrm{~Hz}, 1 \mathrm{H}), 2.07-1.89(\mathrm{~m}, 2 \mathrm{H}), 1.87-1.76$ $(\mathrm{m}, 1 \mathrm{H}), 1.70-1.58(\mathrm{~m}, 1 \mathrm{H}), 1.55-1.44(\mathrm{~m}, 2 \mathrm{H}), 1.23(\mathrm{dd}, J=12.0,6.5 \mathrm{~Hz}, 3 \mathrm{H}), 1.13(\mathrm{dd}, J=$ 10.3, 7.1 Hz, 3H), $\left.1.04(\mathrm{~s}, 9 \mathrm{H}) ;{ }^{13} \mathrm{C} \mathrm{NMR} \mathrm{(125} \mathrm{MHz,} \mathrm{CDCl}_{3}\right) \delta$ (mixture) 174.3, 173.8, 135.8, $135.7,133.7,133.6,133.5,133.4,129.9,129.8,127.8,117.9,117.8,80.7,80.3,76.2,75.1,75.0$, $65.5,65.4,55.4,45.5,42.1,42.0,31.3,28.4,28.2,26.9,26.8,19.4,19.2,13.4,13.2 ;[\alpha]_{\mathrm{D}}+11.6,(\mathrm{c}$ 1.01, $\mathrm{CHCl}_{3}$ ); ESI-HRMS $\mathrm{m} / \mathrm{z}$ calcd for $\mathrm{C}_{30} \mathrm{H}_{42} \mathrm{~N}_{3} \mathrm{O}_{4} \mathrm{Si}[\mathrm{M}+\mathrm{H}]^{+}$536.2939, found 536.2926; ESIHRMS $m / z$ calcd for $\mathrm{C}_{30} \mathrm{H}_{41} \mathrm{~N}_{3} \mathrm{NaO}_{4} \mathrm{Si}[\mathrm{M}+\mathrm{Na}]^{+}$558.2759, found 558.2734.

\section{General procedure for amide preparation}

To a $0.17 \mathrm{M}$ solution of azidoester 20a or $20 \mathbf{b}$ (1.0 eq) in 5:1 mixture of THF and water was added triphenylphosphine $(1.5 \mathrm{eq})$. The reaction mixture was stirred at room temperature for $48 \mathrm{~h}$. The solvent was removed under reduced pressure, the residue was dissolved in $\mathrm{CH}_{2} \mathrm{Cl}_{2}$, dried over $\mathrm{Na}_{2} \mathrm{SO}_{4}$ and concentrated under reduced pressure. $0.1 \mathrm{M}$ solution of the crude amine in anhydrous THF was cooled down to $0{ }^{\circ} \mathrm{C}$. N-methylmorpholine (3.0 eq) was added followed by a dropwise addition of $0.19 \mathrm{M}$ solution of the mixed vinylacetic acid / trimethylacetic acid anhydride in THF (2.0 eq). The reaction mixture was stirred at $0{ }^{\circ} \mathrm{C}$ for $1 \mathrm{hr}$ and at room temperature for another $16 \mathrm{~h}$ under argon. After evaporation of the solvent under reduced pressure, the residue was re-dissolved in an ice-cold 3:1 emulsion of ether and $1.0 \mathrm{M} \mathrm{HCl}(3.0$ eq). After vigorous stirring the organic layer was separated, and the aqueous layer was extracted with ether. The combined organic extracts were dried over $\mathrm{MgSO}_{4}$ and concentrated under reduced pressure. The crude product was purified by flash chromatography $\left(\mathrm{SiO}_{2}, 1: 100\right.$ ratio of the crude product to silica gel, 25\% ether/pentane $\rightarrow$ $30 \%$ ether/pentane).

2-[5-(2-But-3-enoylaminopropyl)tetrahydrofuran-2-yl]propionic 1-(tertbutyldiphenylsilanyloxymethyl)allyl ester (21a). Using the general procedure as described above, 
azidoester 20a $(0.722 \mathrm{~g}, 1.35 \mathrm{mmol})$ was reacted to give $0.639 \mathrm{~g}(82 \%)$ of esteramide 21a as a colorless oil (mixture of diastereomers) after chromatography: TLC $\mathrm{R}_{\mathrm{f}}=0.27$ (60\% ether/pentane); IR (neat, $\mathrm{KBr}, \mathrm{cm}^{-1}$ ) 3294 (broad), 3072, 2961, 2930, 2858, 1736, 1651, 1541, 1462, 1427, 1258, 1190, 1113, 1088, 997, 922; ${ }^{1} \mathrm{H}$ NMR $\left(500 \mathrm{MHz}, \mathrm{CDCl}_{3}\right) \delta$ (mixture) $7.66(\mathrm{dt}, J=7.9,1.3 \mathrm{~Hz}, 4 \mathrm{H})$, $7.45-7.34(\mathrm{~m}, 6 \mathrm{H}), 6.06-5.96$ (two doublets, $1 \mathrm{H}), 5.95-5.85(\mathrm{~m}, 1 \mathrm{H}), 5.85-5.77(\mathrm{~m}, 1 \mathrm{H}), 5.48$ $-5.38(\mathrm{~m}, 1 \mathrm{H}), 5.31(\mathrm{ddt}, J=17.3,8.2,1.2 \mathrm{~Hz}, 1 \mathrm{H}), 5.23-5.13(\mathrm{~m}, 3 \mathrm{H}), 4.05(\mathrm{dq}, J=12.0,7.2$ $\mathrm{Hz}, 1 \mathrm{H}), 4.01-3.88(\mathrm{~m}, 1 \mathrm{H}), 3.87-3.80(\mathrm{~m}, 1 \mathrm{H}), 3.77$ (ddd, $J=10.4,6.7,3.6 \mathrm{~Hz}, 1 \mathrm{H}), 3.71$ (ddd, $J=10.7,6.1,4.5 \mathrm{~Hz}, 1 \mathrm{H}), 2.93(\mathrm{dd}, J=7.1,1.2 \mathrm{~Hz}, 2 \mathrm{H}), 2.59(\mathrm{dp}, J=10.2,7.1 \mathrm{~Hz}, 1 \mathrm{H}), 2.06-$ $1.96(\mathrm{~m}, 1 \mathrm{H}), 1.96-1.86(\mathrm{~m}, 1 \mathrm{H}), 1.69-1.54(\mathrm{~m}, 3 \mathrm{H}), 1.52-1.40(\mathrm{~m}, 1 \mathrm{H}), 1.15(\mathrm{dd}, J=6.1,2.8$ $\mathrm{Hz}, 3 \mathrm{H}), 1.12(\mathrm{dd}, J=8.0,6.2 \mathrm{~Hz}, 3 \mathrm{H}), 1.04(\mathrm{~s}, 9 \mathrm{H}) ;{ }^{13} \mathrm{C}$ NMR $\left(125 \mathrm{MHz}, \mathrm{CDCl}_{3}\right) \delta$ (mixture) $174.0,173.7,170.4,135.6,135.5,133.5,133.4,133.3,133.2,131.8,131.7,129.8,129.7,127.7$, $119.1,119.0,118.0,117.8,80.7,80.4,77.8,77.7,75.0,74.9,65.4,65.3,45.4,45.2,44.7,44.6,42.5$, $42.4,41.8,31.7,31.6,28.1,28.0,26.8,26.7,21.8,19.3,13.4,13.2$; [ $\alpha]_{\mathrm{D}}-9.2$ (c 1.00, $\mathrm{CHCl}_{3}$ ); ESIHRMS m/z calcd for $\mathrm{C}_{34} \mathrm{H}_{48} \mathrm{NO}_{5} \mathrm{Si}[\mathrm{M}+\mathrm{H}]^{+}$578.3296, found 578.3277; ESI-HRMS m/z calcd for $\mathrm{C}_{34} \mathrm{H}_{47} \mathrm{NNaO}_{5} \mathrm{Si}[\mathrm{M}+\mathrm{Na}]^{+} 600.3116$, found 600.3101 .

2-[5-(2-But-3-enoylaminopropyl)tetrahydrofuran-2-yl]propionic

acid

1-(tertbutyldiphenylsilanyloxymethyl)allyl ester (21b). Using the general procedure as described above, azidoester 20b $(0.163 \mathrm{~g}, 0.31 \mathrm{mmol})$ was reacted to give $0.1135 \mathrm{~g}(64 \%)$ of esteramide $21 \mathrm{~b}$ as a colorless oil (mixture of diastereomers) after chromatography: TLC $\mathrm{R}_{\mathrm{f}}=0.27$ (60\% ether/pentane); IR (neat, $\mathrm{KBr}, \mathrm{cm}^{-1}$ ) 3294 (broad), 2959, 2930, 2856, 2359, 1732, 1651, 1549, 1462, 1427, 1258, 1190, 1113, 1088, 997, 922; ${ }^{1} \mathrm{H}$ NMR $\left(500 \mathrm{MHz}, \mathrm{CDCl}_{3}\right) \delta 7.70-7.64(\mathrm{~m}, 4 \mathrm{H}), 7.46-7.35(\mathrm{~m}$, $6 \mathrm{H}), 6.00-5.85(\mathrm{~m}, 2 \mathrm{H}), 5.85-5.77(\mathrm{~m}, 1 \mathrm{H}), 5.49-5.39(\mathrm{~m}, 1 \mathrm{H}), 5.32(\mathrm{ddt}, J=17.0,8.2,1.2 \mathrm{~Hz}$, $1 \mathrm{H}), 5.24-5.18(\mathrm{~m}, 2 \mathrm{H}), 5.18-5.13(\mathrm{~m}, 1 \mathrm{H}), 4.06(\mathrm{dq}, J=11.6,7.2 \mathrm{~Hz}, 1 \mathrm{H}), 4.01-3.88(\mathrm{~m}, 1 \mathrm{H})$, $3.88-3.80(\mathrm{~m}, 1 \mathrm{H}), 3.76(\mathrm{ddd}, J=10.4,6.7,3.5 \mathrm{~Hz}, 1 \mathrm{H}), 3.71(\mathrm{ddd}, J=10.8,6.3,4.5 \mathrm{~Hz}, 1 \mathrm{H})$, $2.93(\mathrm{dd}, J=7.1,1.2 \mathrm{~Hz}, 2 \mathrm{H}), 2.59(\mathrm{dp}, J=10.1,7.1 \mathrm{~Hz}, 1 \mathrm{H}), 2.08-1.97(\mathrm{~m}, 1 \mathrm{H}), 1.97-1.86(\mathrm{~m}$, $1 \mathrm{H}), 1.70-1.53(\mathrm{~m}, 3 \mathrm{H}), 1.53-1.41(\mathrm{~m}, 1 \mathrm{H}), 1.30-1.19(\mathrm{~m}, 1 \mathrm{H}), 1.15(\mathrm{dd}, J=6.4,2.8 \mathrm{~Hz}, 3 \mathrm{H})$, $1.13(\mathrm{dd}, J=8.4,6.8 \mathrm{~Hz}, 3 \mathrm{H}), 1.04(\mathrm{~s}, 9 \mathrm{H}) ;{ }^{13} \mathrm{C} \mathrm{NMR}\left(125 \mathrm{MHz}, \mathrm{CDCl}_{3}\right) \delta 174.1,173.7,170.4$, $135.7,135.6,133.7,133.5,133.4,133.3,131.8,131.7,129.9,129.8,127.8,119.3,119.2,118.1$, $117.9,80.7,80.5,77.9,77.8,75.1,75.0,65.5,65.4,45.5,45.3,44.8,44.7,42.5,42.4,41.9,31.8$, 31.7, 28.2, 28.1, 26.9, 26.8, 21.9, 19.3, 13.5, 13.2; $[\alpha]_{\mathrm{D}}+6.7$, (c 1.18, $\left.\mathrm{CHCl}_{3}\right)$; ESI-HRMS m/z calcd for $\mathrm{C}_{34} \mathrm{H}_{48} \mathrm{NO}_{5} \mathrm{Si}[\mathrm{M}+\mathrm{H}]^{+}$578.3296, found 578.3278; ESI-HRMS m/z calcd for $\mathrm{C}_{34} \mathrm{H}_{47} \mathrm{NNaO}_{5} \mathrm{Si}$ $[\mathrm{M}+\mathrm{Na}]^{+} 600.3116$, found 600.3098 .

\section{General procedure for ring-closing metathesis}

To a $0.0015 \mathrm{M}$ solution of esteramide 21a or $21 \mathbf{b}$ (1.0 eq) in anhydrous $\mathrm{CH}_{2} \mathrm{Cl}_{2}$ Grubbs'-Hoveyda 2nd generation catalyst (15 mol\%) was added. The reaction mixture was stirred at room temperature under argon for $72 \mathrm{~h}$ until TLC showed no starting material. Ethyl vinyl ether (15.0 eq) was added and the reaction mixture was stirred vigorously for $30 \mathrm{~min}$. Evaporation of the solvent gave the crude product as a mixture of 2 diastereomers that were separated by fllash chromatography $\left(\mathrm{SiO}_{2}\right.$, $1: 100$ ratio of the crude product to silica gel, $27 \%$ ether/pentane $\rightarrow 35 \%$ ether/pentane). In order to remove ruthenium byproducts, each diastereomer was dissolved in minimal amount of ether and treated with activated carbon (Darco ${ }^{\circledR}$ G-60, 100 mesh, powder, 10 wt. eq). The resulting 
suspension was stirred at room temperature for $12 \mathrm{~h}$, filtered through a short plug of Celite and concentrated under reduced pressure.

Using the general procedure as described above, esteramide 21a $(0.616 \mathrm{~g}, 1.07 \mathrm{mmol})$ was reacted to give macrocycles 22a $(0.196 \mathrm{~g}, 33 \%)$ and 22b $(0.203 \mathrm{~g}, 35 \%)$ as colorless oils after chromatography and activated carbon treatment:

$(1 R, 2 R, 5 S, 11 S, 13 S)-5$-(tert-Butyldiphenylsilanyloxymethyl)-2,11-dimethyl-4,16-dioxa-10azabicyclo[11.2.1]hexadec-6-ene-3,9-dione (22a). TLC $\mathrm{R}_{\mathrm{f}}=0.24$ (60\% ether/pentane); IR (neat, $\mathrm{KBr}, \mathrm{cm}^{-1}$ ) 3394, 2959, 2932, 2858, 1732, 1661, 1514, 1462, 1427, 1263, 1113, 824, 743, 704, 505; ${ }^{1} \mathrm{H}$ NMR (500 MHz, $\left.\mathrm{CDCl}_{3}\right) \delta$ (major) $7.71-7.61(\mathrm{~m}, 4 \mathrm{H}), 7.46-7.33(\mathrm{~m}, 6 \mathrm{H}), 6.52(\mathrm{~d}, J=3.4$ $\mathrm{Hz}, 1 \mathrm{H}), 5.98(\mathrm{dt}, J=15.4,7.6 \mathrm{~Hz}, 1 \mathrm{H}), 5.74(\mathrm{dd}, J=15.6,6.9 \mathrm{~Hz}, 1 \mathrm{H}), 5.58-5.53(\mathrm{~m}, 1 \mathrm{H}), 3.88$ $-3.75(\mathrm{~m}, 5 \mathrm{H}), 2.93(\mathrm{~d}, J=7.5 \mathrm{~Hz}, 2 \mathrm{H}), 2.43-2.32(\mathrm{~m}, 1 \mathrm{H}), 2.09-1.82(\mathrm{~m}, 2 \mathrm{H}), 1.77(\mathrm{dd}, J=$ 15.0, 3.0 Hz, 1H), $1.63-1.40(\mathrm{~m}, 3 \mathrm{H}), 1.19(\mathrm{~d}, J=6.2 \mathrm{~Hz}, 3 \mathrm{H}), 1.11(\mathrm{~d}, J=6.9 \mathrm{~Hz}, 3 \mathrm{H}), 1.04(\mathrm{~s}$, $9 \mathrm{H}) ;{ }^{13} \mathrm{C} \mathrm{NMR}\left(125 \mathrm{MHz}, \mathrm{CDCl}_{3}\right) \delta$ (major) 175.4, 170.1, 135.7, 135.6, 133.4, 133.3, 132.0, 129.9, 129.8, 129.7, 127.8, 83.6, 79.4, 72.7, 64.7, 53.5, 47.7, 45.8, 42.3, 40.6, 38.4, 32.0, 29.0, 26.8, 21.5, 19.3, 14.2; $[\alpha]_{\mathrm{D}}+28.0,\left(\mathrm{c} 1.00, \mathrm{CHCl}_{3}\right)$; ESI-HRMS m/z calcd for $\mathrm{C}_{32} \mathrm{H}_{44} \mathrm{NO}_{5} \mathrm{Si}[\mathrm{M}+\mathrm{H}]^{+} 550.2983$, found 550.2968 .

$(1 R, 2 R, 5 S, 11 R, 13 S)-5$-(tert-Butyldiphenylsilanyloxymethyl)-2,11-dimethyl-4,16-dioxa-10azabicyclo[11.2.1]hexadec-6-ene-3,9-dione (22b). TLC $\mathrm{R}_{\mathrm{f}}=0.18(60 \%$ ether/pentane); IR (neat, $\mathrm{KBr}, \mathrm{cm}^{-1}$ ) 3387, 2959, 2930, 2858, 1732, 1666, 1520, 1462, 1427, 1248, 1113, 1055, 824, 743, 704, 505; ${ }^{1} \mathrm{H}$ NMR (500 MHz, $\left.\mathrm{CDCl}_{3}\right) \delta 7.69-7.62(\mathrm{~m}, 4 \mathrm{H}), 7.45-7.33(\mathrm{~m}, 6 \mathrm{H}), 6.67(\mathrm{~d}, J=4.1$ $\mathrm{Hz}, 1 \mathrm{H}), 5.91(\mathrm{dt}, J=15.3,7.6 \mathrm{~Hz}, 1 \mathrm{H}), 5.63(\mathrm{dd}, J=15.5,7.6 \mathrm{~Hz}, 1 \mathrm{H}), 5.36$ (td, $J=7.2,3.8 \mathrm{~Hz}$, $1 \mathrm{H}), 4.05$ (qd, $J=7.6,3.7 \mathrm{~Hz}, 1 \mathrm{H}), 3.85-3.77(\mathrm{~m}, 2 \mathrm{H}), 3.74(\mathrm{dd}, J=11.2,3.8 \mathrm{~Hz}, 2 \mathrm{H}), 2.96(\mathrm{dd}, J$ $=17.2,7.6 \mathrm{~Hz}, 1 \mathrm{H}), 2.91(\mathrm{dd}, J=17.2,7.8 \mathrm{~Hz}, 1 \mathrm{H}), 2.32(\mathrm{dq}, J=10.4,6.9 \mathrm{~Hz}, 1 \mathrm{H}), 2.04-1.92$ $(\mathrm{m}, 2 \mathrm{H}), 1.82(\mathrm{dd}, J=14.8,3.4 \mathrm{~Hz}, 1 \mathrm{H}), 1.70-1.61(\mathrm{~m}, 1 \mathrm{H}), 1.57-1.45(\mathrm{~m}, 2 \mathrm{H}), 1.17(\mathrm{~d}, J=6.2$ $\mathrm{Hz}, 3 \mathrm{H}), 1.14(\mathrm{~d}, J=6.9 \mathrm{~Hz}, 3 \mathrm{H}), 1.03(\mathrm{~s}, 9 \mathrm{H}) ;{ }^{13} \mathrm{C} \mathrm{NMR}\left(125 \mathrm{MHz}, \mathrm{CDCl}_{3}\right) \delta 173.3,170.3,135.6$, $135.5,133.3,133.2,131.6,129.9,129.8,128.8,127.8,127.7,82.1,80.2,74.2,64.9,53.5$, 48.7, 47.5, 41.9, 40.2, 31.8, 28.7, 26.7, 21.7, 19.23, 13.9; $[\alpha]_{\mathrm{D}}+18.8,\left(\mathrm{c} 1.00, \mathrm{CHCl}_{3}\right)$; ESI-HRMS m/z calcd for $\mathrm{C}_{32} \mathrm{H}_{44} \mathrm{NO}_{5} \mathrm{Si}[\mathrm{M}+\mathrm{H}]^{+} 550.2983$, found 550.2967.

Using the general procedure as described above, esteramide $21 \mathbf{b}(0.6169 \mathrm{~g}, 1.07 \mathrm{mmol})$ was reacted to give esteramide 23a $(0.037 \mathrm{~g}, 50 \%)$ and $23 \mathrm{~b}(0.023 \mathrm{~g}, 32 \%)$ as colorless oils after chromatography and activated carbon treatment:

(1R,2R,5R,11S,13S)-5-(tert-Butyldiphenylsilanyloxymethyl)-2,11-dimethyl-4,16-dioxa-10azabicyclo[11.2.1]hexadec-6-ene-3,9-dione (23a). TLC $\mathrm{R}_{\mathrm{f}}=0.25$ (60\% ether/pentane); IR (neat, $\left.\mathrm{KBr}, \mathrm{cm}^{-1}\right)$ 3394, 2961, 2932, 2858, 1732, 1661, 1516, 1462, 1427, 1371, 1267, 1190, 1169, 1134, 1113, 1072, 1030, 976, 824, 739, 704, 505; ${ }^{1} \mathrm{H}$ NMR $\left(500 \mathrm{MHz}, \mathrm{CDCl}_{3}\right) \delta$ (major) $7.73-7.61(\mathrm{~m}$, 4H), $7.48-7.34(\mathrm{~m}, 6 \mathrm{H}), 6.52(\mathrm{~d}, J=3.1 \mathrm{~Hz}, 1 \mathrm{H}), 5.98(\mathrm{dt}, J=15.4,7.6 \mathrm{~Hz}, 1 \mathrm{H}), 5.74(\mathrm{dd}, J=$ $15.6,6.9 \mathrm{~Hz}, 1 \mathrm{H}), 5.56(\mathrm{td}, J=6.8,3.9 \mathrm{~Hz}, 1 \mathrm{H}), 3.89-3.80(\mathrm{~m}, 2 \mathrm{H}), 3.78(\mathrm{dd}, J=11.2,4.2 \mathrm{~Hz}$, $1 \mathrm{H}), 3.76-3.67(\mathrm{~m}, 1 \mathrm{H}), 2.94(\mathrm{~d}, J=7.6 \mathrm{~Hz}, 2 \mathrm{H}), 2.39(\mathrm{dq}, J=10.0,6.9 \mathrm{~Hz}, 1 \mathrm{H}), 2.10-1.94(\mathrm{~m}$, 2H), $1.77(\mathrm{dd}, J=15.0,3.0 \mathrm{~Hz}, 1 \mathrm{H}), 1.63-1.35(\mathrm{~m}, 4 \mathrm{H}), 1.20(\mathrm{~d}, J=6.2 \mathrm{~Hz}, 3 \mathrm{H}), 1.11(\mathrm{~d}, J=6.9$ $\mathrm{Hz}, 3 \mathrm{H}), 1.05(\mathrm{~s}, 9 \mathrm{H}) ;{ }^{13} \mathrm{C} \mathrm{NMR}\left(125 \mathrm{MHz}, \mathrm{CDCl}_{3}\right) \delta$ (major) 175.5, 170.3, 135.7, 133.4, 132.1, $129.9,127.8,125.4,83.7,79.5,72.4,64.7,47.8,45.9,42.4,40.6,32.1,29.1,26.9,21.6,19.4,14.3$; 
$[\alpha]_{\mathrm{D}}-11.7$ (c 1.01, $\mathrm{CHCl}_{3}$ ); ESI-HRMS m/z calcd for $\mathrm{C}_{32} \mathrm{H}_{44} \mathrm{NO}_{5} \mathrm{Si}[\mathrm{M}+\mathrm{H}]^{+}$550.2983, found 550.2968; ESI-HRMS m/z calcd for $\mathrm{C}_{32} \mathrm{H}_{43} \mathrm{NNaO}_{5} \mathrm{Si}[\mathrm{M}+\mathrm{Na}]^{+}$572.2803, found 572.2788.

$(1 R, 2 R, 5 R, 11 R, 13 S)-5$-(tert-Butyldiphenylsilanyloxymethyl)-2,11-dimethyl-4,16-dioxa-10-

azabicyclo[11.2.1]hexadec-6-ene-3,9-dione (23b). TLC $\mathrm{R}_{\mathrm{f}}=0.20$ (60\% ether/pentane); IR (neat, $\left.\mathrm{KBr}, \mathrm{cm}^{-1}\right)$ 3385, 2959, 2932, 2858, 2361, 2341, 1734, 1663, 1522, 1458, 1427, 1250, 1113, 1055 , 1030, 976, 744, 704, 505; ${ }^{1} \mathrm{H}$ NMR (500 MHz, $\left.\mathrm{CDCl}_{3}\right) \delta 7.70-7.62(\mathrm{~m}, 4 \mathrm{H}), 7.47-7.34(\mathrm{~m}, 6 \mathrm{H})$, $6.67(\mathrm{~d}, J=4.0 \mathrm{~Hz}, 1 \mathrm{H}), 5.91(\mathrm{dt}, J=15.3,7.6 \mathrm{~Hz}, 1 \mathrm{H}), 5.64(\mathrm{dd}, J=15.5,7.6 \mathrm{~Hz}, 1 \mathrm{H}), 5.37(\mathrm{td}, J$ $=7.2,3.8 \mathrm{~Hz}, 1 \mathrm{H}), 4.05(\mathrm{ddd}, J=10.3,7.7,2.8 \mathrm{~Hz}, 1 \mathrm{H}), 3.87-3.77(\mathrm{~m}, 2 \mathrm{H}), 3.74(\mathrm{dd}, J=11.2$, $3.8 \mathrm{~Hz}, 2 \mathrm{H}), 2.97(\mathrm{dd}, J=17.2,7.6 \mathrm{~Hz}, 1 \mathrm{H}), 2.92(\mathrm{dd}, J=17.4,8.0 \mathrm{~Hz}, 1 \mathrm{H}), 2.33(\mathrm{dq}, J=10.5,6.9$ $\mathrm{Hz}, 1 \mathrm{H}), 2.06-1.93(\mathrm{~m}, 2 \mathrm{H}), 1.82(\mathrm{dd}, J=14.8,3.4 \mathrm{~Hz}, 1 \mathrm{H}), 1.70-1.63(\mathrm{~m}, 1 \mathrm{H}), 1.58-1.43(\mathrm{~m}$, $2 \mathrm{H}), 1.18(\mathrm{~d}, J=6.2 \mathrm{~Hz}, 3 \mathrm{H}), 1.15(\mathrm{~d}, J=6.9 \mathrm{~Hz}, 3 \mathrm{H}), 1.04(\mathrm{~s}, 9 \mathrm{H}) ;{ }^{13} \mathrm{C} \mathrm{NMR}\left(125 \mathrm{MHz}, \mathrm{CDCl}_{3}\right) \delta$ $173.4,170.4,135.7,135.6,133.4,133.3,131.7,129.9,129.8,128.9,127.9,127.8,82.2,80.3,74.3$, $65.0,48.8,47.6,42.0,40.3,31.9,28.8,26.8,21.8,19.4,14.0 ;[\alpha]_{\mathrm{D}}-18.1$ (c 1.03, $\left.\mathrm{CHCl}_{3}\right)$; ESIHRMS $\mathrm{m} / \mathrm{z}$ calcd for $\mathrm{C}_{32} \mathrm{H}_{44} \mathrm{NO}_{5} \mathrm{Si}[\mathrm{M}+\mathrm{H}]^{+}$550.2983, found 550.2992; ESI-HRMS m/z calcd for $\mathrm{C}_{32} \mathrm{H}_{43} \mathrm{NNaO}_{5} \mathrm{Si}[\mathrm{M}+\mathrm{Na}]^{+}$572.2803, found 572.2816.

\section{General procedure for TBDPS deprotection}

To a $0.1 \mathrm{M}$ solution of TBDPS protected alcohol (1.0 eq) in THF was added a solution of HF in pyridine (20.0 eq, $70 \%$ by weight) in one portion. The resulting solution was stirred at room temperature for $72 \mathrm{~h}$ until TLC showed no starting material. Methoxytrimethylsilane (60 eq) was added, and the reaction mixture was stirred for $10 \mathrm{~min}$ to neutralize the excess of HF. After evaporation of the solvent under reduced pressure, the crude product was purified by flash chromatography $\left(\mathrm{SiO}_{2}, 1: 50\right.$ ratio of crude product to silica gel, $60 \%$ ether/hexanes $\rightarrow 100 \%$ ether).

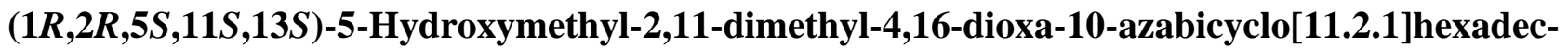
6-ene-3,9-dione (24a). Using the general procedure as described above, compound 22a (0.141 g, $0.26 \mathrm{mmol})$ was reacted to give alcohol 24a as a colorless oil $(0.065 \mathrm{~g}, 82 \%)$ after flash chromatography $\left(\mathrm{SiO}_{2}, 7 \mathrm{~g}, 60 \%\right.$ ether/hexanes $\rightarrow$ 80\% ether/hexanes): TLC $\mathrm{R}_{\mathrm{f}}=0.16(100 \%$ ether); IR (neat, $\mathrm{KBr}, \mathrm{cm}^{-1}$ ) 3383, 3304 (broad), 2968, 2935, 2876, 1728, 1651, 1531, 1454, 1373, 1269, 1192, 1167, 1097, 1070, 1038, 976; ${ }^{1} \mathrm{H}$ NMR $\left(500 \mathrm{MHz}, \mathrm{CDCl}_{3}\right) \delta$ (major) $6.53(\mathrm{~d}, J=2.9$ $\mathrm{Hz}, 1 \mathrm{H}), 5.99$ (dt, $J=15.3,7.5 \mathrm{~Hz}, 1 \mathrm{H}), 5.85(\mathrm{dd}, J=15.7,6.9 \mathrm{~Hz}, 1 \mathrm{H}), 5.44(\mathrm{dd}, J=11.8,5.7 \mathrm{~Hz}$, 1H), $3.90-3.65(\mathrm{~m}, 4 \mathrm{H}), 2.98(\mathrm{dd}, J=16.7,8.0 \mathrm{~Hz}, 1 \mathrm{H}), 2.92(\mathrm{dd}, J=16.6,7.1 \mathrm{~Hz}, 1 \mathrm{H}), 2.84-$ $2.74(\mathrm{~m}, 1 \mathrm{H}), 2.71(\mathrm{bs}, 1 \mathrm{H}), 2.40(\mathrm{dq}, J=10.3,7.1 \mathrm{~Hz}, 1 \mathrm{H}), 2.07-1.89(\mathrm{~m}, 2 \mathrm{H}), 1.75(\mathrm{dd}, J=$ 15.1, 3.2 Hz, 1H), $1.60-1.34(\mathrm{~m}, 3 \mathrm{H}), 1.17(\mathrm{~d}, J=6.1 \mathrm{~Hz}, 3 \mathrm{H}), 1.10(\mathrm{~d}, J=6.8 \mathrm{~Hz}, 3 \mathrm{H}) ;{ }^{13} \mathrm{C}$ NMR $\left(125 \mathrm{MHz} \mathrm{CDCl}_{3}\right) \delta$ (major) 175.5, 170.3, 131.9, 130.0, 83.6, 79.5, 72.9, 63.5, 47.8, 45.7, 42.2, 40.5, 32.0, 29.0, 21.5, 14.4; $[\alpha]_{\mathrm{D}}+49.0$, (c 1.00, $\left.\mathrm{CHCl}_{3}\right)$; ESI-HRMS m/z calcd for $\mathrm{C}_{16} \mathrm{H}_{26} \mathrm{NO}_{5}[\mathrm{M}+$ $\mathrm{H}]^{+} 312.1805$, found 312.1796 .

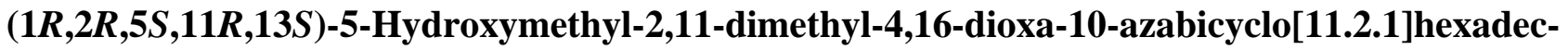
6-ene-3,9-dione (24b). Using the general procedure as described above, compound $22 \mathbf{b}(0.148 \mathrm{~g}$, $0.27 \mathrm{mmol})$ was reacted to give alcohol $\mathbf{2 4 b}$ as a white solid $(0.064 \mathrm{~g}, 76 \%)$ after flash chromatography $\left(\mathrm{SiO}_{2}, 8 \mathrm{~g}, 80 \%\right.$ ether/hexanes $\rightarrow 100 \%$ ether): $\mathrm{mp} 152.4-157.2{ }^{\circ} \mathrm{C}$ (decomp.), TLC $\mathrm{R}_{\mathrm{f}}=0.08$ (100\% ether); IR (neat, $\mathrm{KBr}, \mathrm{cm}^{-1}$ ) 3423 (broad), 3377, 2966, 2934, 2876, 1732, 1645, 
1531, 1252, 1090, 1072, 1057, 1028, 976; ${ }^{1} \mathrm{H} \mathrm{NMR}\left(500 \mathrm{MHz}, \mathrm{CDCl}_{3}\right) \delta 6.65(\mathrm{~d}, J=3.9 \mathrm{~Hz}, 1 \mathrm{H})$, $5.94(\mathrm{dt}, J=15.3,7.6 \mathrm{~Hz}, 1 \mathrm{H}), 5.65(\mathrm{dd}, J=15.6,7.7 \mathrm{~Hz}, 1 \mathrm{H}), 5.29$ (dt, $J=7.5,5.5 \mathrm{~Hz}, 1 \mathrm{H}), 4.02$ $(\mathrm{ddd}, J=10.3,7.6,2.7 \mathrm{~Hz}, 1 \mathrm{H}), 3.80(\mathrm{td}, J=10.0,4.9 \mathrm{~Hz}, 1 \mathrm{H}), 3.72(\mathrm{~d}, J=5.1 \mathrm{~Hz}, 2 \mathrm{H}$, overlapping), $3.77-3.67$ (m, 1H, overlapping), 2.98 (dd, $J=15.1,5.4 \mathrm{~Hz}, 1 \mathrm{H}), 2.93$ (dd, $J=15.0$, $5.7 \mathrm{~Hz}, 1 \mathrm{H}), 2.53(\mathrm{bs}, 1 \mathrm{H}), 2.30(\mathrm{dq}, J=10.4,6.9 \mathrm{~Hz}, 1 \mathrm{H}), 2.02-1.90(\mathrm{~m}, 2 \mathrm{H}), 1.80(\mathrm{dd}, J=14.8$, $3.4 \mathrm{~Hz}, 1 \mathrm{H}), 1.68-1.59(\mathrm{~m}, 1 \mathrm{H}), 1.55-1.41(\mathrm{~m}, 2 \mathrm{H}), 1.15(\mathrm{~d}, J=6.2 \mathrm{~Hz}, 3 \mathrm{H}), 1.12(\mathrm{~d}, J=6.9 \mathrm{~Hz}$, $3 \mathrm{H}) ;{ }^{13} \mathrm{C}$ NMR $\left(125 \mathrm{MHz}, \mathrm{CDCl}_{3}\right) \delta 173.2,170.3,131.4,129.4,82.1,80.3,74.4,63.8,48.7,47.6$, 42.0, 40.2 , 31.8, 28.7, 21.7, 14.0; $[\alpha]_{\mathrm{D}}+61.3,\left(\mathrm{c} 1.00, \mathrm{CHCl}_{3}\right)$; ESI-HRMS m/z calcd for $\mathrm{C}_{16} \mathrm{H}_{26} \mathrm{NO}_{5}$ $[\mathrm{M}+\mathrm{H}]^{+} 312.1805$, found 312.1789 .

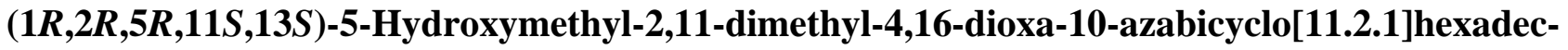
6-ene-3,9-dione (25a). Using the general procedure as described above, compound 23a (0.035 g, $0.06 \mathrm{mmol})$ was reacted to give alcohol 25a as a colorless oil $(0.016 \mathrm{~g}, 79 \%)$ after flash chromatography ( $\mathrm{SiO}_{2}, 2 \mathrm{~g}, 60 \%$ ether/hexanes $\rightarrow 80 \%$ ether/hexanes): IR (neat, $\mathrm{KBr}, \mathrm{cm}^{-1}$ ) 3385 (broad), 3329, 2968, 2937, 2876, 2361, 2343, 1728, 1647, 1527, 1375, 1165, 1070, 1038, 976; ${ }^{1} \mathrm{H}$ NMR (500 MHz, $\left.\mathrm{CDCl}_{3}\right) \delta$ (major) $6.53(\mathrm{~d}, J=2.5 \mathrm{~Hz}, 1 \mathrm{H}), 6.04(\mathrm{dt}, J=15.3,7.6 \mathrm{~Hz}, 1 \mathrm{H}), 5.88$ $(\mathrm{dd}, J=15.7,6.9 \mathrm{~Hz}, 1 \mathrm{H}), 5.47(\mathrm{dd}, J=11.8,5.4 \mathrm{~Hz}, 1 \mathrm{H}), 3.93-3.82(\mathrm{~m}, 2 \mathrm{H}), 3.81(\mathrm{~d}, J=5.3 \mathrm{~Hz}$, 2H), $3.80-3.69(\mathrm{~m}, 1 \mathrm{H}), 3.02(\mathrm{dd}, J=16.6,7.6 \mathrm{~Hz}, 1 \mathrm{H}), 2.96(\mathrm{dd}, J=16.6,7.6 \mathrm{~Hz}, 1 \mathrm{H}) ; 2.43$ (dq, $J=9.9,6.9 \mathrm{~Hz}, 1 \mathrm{H}), 2.10-1.94(\mathrm{~m}, 2 \mathrm{H}), 1.79(\mathrm{dd}, J=15.1,3.2 \mathrm{~Hz}, 1 \mathrm{H}$, overlapping), $1.94-1.71$ (m, 1H, overlapping), $1.55-1.39(\mathrm{~m}, 3 \mathrm{H}), 1.21(\mathrm{~d}, J=6.1 \mathrm{~Hz}, 3 \mathrm{H}), 1.14(\mathrm{~d}, J=6.9 \mathrm{~Hz}, 3 \mathrm{H}) ;{ }^{13} \mathrm{C}$ NMR $\left(125 \mathrm{MHz}, \mathrm{CDCl}_{3}\right) \delta$ (major) $175.5,170.1,131.5,130.5,83.6,79.6,73.0,63.7,47.8,45.8$, 42.3, 40.7 32.0, 29.1, 21.6, 14.5; [ $\alpha]_{\mathrm{D}}$-29.4, (c 1.00, $\mathrm{CHCl}_{3}$ ); ESI-HRMS m/z calcd for $\mathrm{C}_{16} \mathrm{H}_{26} \mathrm{NO}_{5}$ $[\mathrm{M}+\mathrm{H}]^{+}$312.1805, found 312.1815; ESI-HRMS m/z calcd for $\mathrm{C}_{16} \mathrm{H}_{25} \mathrm{NNaO}_{5}[\mathrm{M}+\mathrm{Na}]^{+} 334.1625$, found 334.1637 .

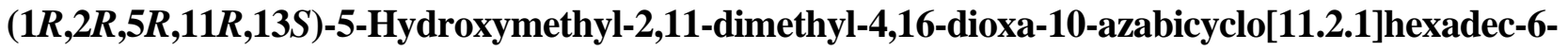

ene-3,9-dione (25b). Using the general procedure as described above, compound 23b $(0.020 \mathrm{~g}, 0.04$ mmol) was reacted to give alcohol 63b as a colorless oil (0.009 g, 80\%) after flash chromatography $\left(\mathrm{SiO}_{2}, 1 \mathrm{~g}, 80 \%\right.$ ether/hexanes $\rightarrow 100 \%$ ether): IR (neat, $\mathrm{KBr}, \mathrm{cm}^{-1}$ ) 3381, 3325 (broad), 2924, 2853, 2359, 2332, 1732, 1645, 1531, 1462, 1377, 1261, 1169, 1086, 1074, 976, 959; ${ }^{1} \mathrm{H}$ NMR (500 $\left.\mathrm{MHz} \mathrm{CDCl}_{3}\right) \delta 6.68(\mathrm{~d}, J=3.4 \mathrm{~Hz}, 1 \mathrm{H}), 5.97(\mathrm{dt}, J=15.3,7.6 \mathrm{~Hz}, 1 \mathrm{H}), 5.67(\mathrm{dd}, J=15.5,7.7 \mathrm{~Hz}$, $1 \mathrm{H}), 5.31$ (dt, $J=8.3,4.2 \mathrm{~Hz}, 1 \mathrm{H}), 4.05$ (ddd, $J=10.3,7.7,2.7 \mathrm{~Hz}, 1 \mathrm{H}), 3.84$ (ddd, $J=15.5,7.1$, $3.8 \mathrm{~Hz}, 1 \mathrm{H}), 3.80-3.70(\mathrm{~m}, 1 \mathrm{H}$, overlapping), $3.75(\mathrm{~d}, J=5.5 \mathrm{~Hz}, 2 \mathrm{H}$, overlapping), $3.02(\mathrm{dd}, J=$ 15.2, $5.5 \mathrm{~Hz}, 1 \mathrm{H}), 2.98(\mathrm{dd}, J=14.9,5.7 \mathrm{~Hz}, 1 \mathrm{H}), 2.33(\mathrm{dq}, J=10.5,6.9 \mathrm{~Hz}, 1 \mathrm{H}), 2.04-1.94(\mathrm{~m}$, 2H), 1.88 (bs, 1H), 1.83 (dd, $J=14.9,3.4 \mathrm{~Hz}, 1 \mathrm{H}), 1.70-1.63(\mathrm{~m}, 1 \mathrm{H}), 1.57-1.46(\mathrm{~m}, 2 \mathrm{H}), 1.18$ $(\mathrm{d}, J=6.2 \mathrm{~Hz}, 3 \mathrm{H}), 1.15(\mathrm{~d}, J=6.9 \mathrm{~Hz}, 3 \mathrm{H}) ;{ }^{13} \mathrm{C} \mathrm{NMR}\left(125 \mathrm{MHz}, \mathrm{CDCl}_{3}\right) \delta 173.3,170.6,131.2$,

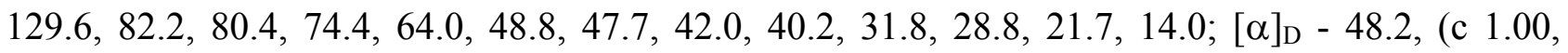
$\mathrm{CHCl}_{3}$ ); ESI-HRMS m/z calcd for $\mathrm{C}_{16} \mathrm{H}_{26} \mathrm{NO}_{5}[\mathrm{M}+\mathrm{H}]^{+}$312.1805, found 312.1810; ESI-HRMS m/z calcd for $\mathrm{C}_{16} \mathrm{H}_{26} \mathrm{NNaO}_{5}[\mathrm{M}+\mathrm{Na}]^{+} 334.1625$, found 334.1632. 


\section{Acknowledgements}

The authors would like to thank the National Institutes of Health (NIGMS R42 FM-07215) for generous support of this work.

\section{References}

1. Villar, H.O.; Hansen, M. R. Exp. Opinion Drug Disc. 2009, 4, 1215.

2. (a) Cordier, C.; Morton, D.; Murrison, S.; Nelson, A.; O’Leray-Steele, C. Nat. Prod. Rep. 2008, 4, 719.

(b) Shang, S.; Tan, D. S. Curr. Opinion Chem. Biol. 2005, 3, 248.

3. (a) Earle, M. J.; Priestley, N. D. Bioorg. Med. Chem. Lett. 1997, 7, 2187. (b) Walczak, R. J.; Nelson, M. E.; Priestley, N. D. J. Am. Chem. Soc. 2001, 123, 10415. (c) Walczak, R. J.; Woo, A. J.; Strohl, W. R.; Priestley, N. D. FEMS Lett. 2000, 183, 171.

4. Nikodinovic, J.; Dinges, J. M.; Bergmeier, S. C.; McMills, M. C.; Wright, D. L.; Priestley, N. D. Org. Lett. 2006, 8, 443.

5. (a) Ashworth, D. M.; Clark, C. A.; Robinson, J. A. J. Chem. Soc., Perkin Trans. 1 1989, 1461.

(b) Dinges, J. M.; Bessette Jr., B. A.; Cox, J. E.; Redder, C. R.; Priestley, N. D. Biotechnol. Prog. 2006, 22, 135.

6. Randolph, J. T.; Wald, P.; Nichols, C.; Sauer, D.; Haviv, F.; Diaz, G.; Bammert, G.; Besecke, L. M.; Segreti, J. A.; Mohning, K. M.; Bush, E. N.; Wegner, C. D.; Greer, J., J. Med. Chem. 2004, 47, 1085.

7. Ma, Z.; Clark, R. F.; Brazzle, A.; Wang, S.; Rupp, M. J.; Li, I.; Griesgraber, G.; Zhang, S.; Yong, H.; Phan, L. T.; Nemoto, P. A.; Chu, D. T. W.; Plattner, J. J.; Zhang, X.; Zhong, P.; Cao, Z.; Nilius, A. M.; Shortridge, V. D.; Flamm, R. K.; Mitten, M.; Muelbroek, J.; Ewing, P.; Alder, J.; Or, Y. S. J. Med. Chem. 2001, 44, 4137.

8. Fleming, I.; Ghosh, S. K. J. Chem. Soc., Perkin Trans. 1 1998, 2733.

9. (a) Young, W. G.; Prater, A. N.; Winstein, S. J. Am. Chem. Soc. 1933, 55, 4908. (b) Lane, J. F.; Roberts, J. D.; Young, W. G. J. Am. Chem. Soc. 1944, 66, 543.

10. Crosignani, S.; Gonzalez, J.; Swinnen, D. Org. Lett. 2004, 6, 4579.

11. Rajendra, G.; Miller, M. J. J. Org. Chem. 1987, 52, 4471.

12. Trost, B. M.; McEachern, E. J.; Toste, F. D. J. Am. Chem. Soc. 1998, 120, 12702.

13. (a) Dobarro, A.; Velasco, D. Tetrahedron 1996, 52, 13525. (b) Dobarro, A.; Velasco, D. Tetrahedron 1996, 52, 13733. 